\title{
Update of the Culicoides (Diptera: Ceratopogonidae) species checklist from Algeria with 10 new records
}

\author{
Mounira Belkharchouche ${ }^{1,2,3,4^{*}}$, Selima Berchi ${ }^{3}$, Bruno Mathieu ${ }^{5}$, Ignace Rakotoarivony ${ }^{4,6}$, Maxime Duhayon ${ }^{4,6}$, \\ Thierry Baldet ${ }^{6,7}$ and Thomas Balenghien ${ }^{6,8,9^{*}}$
}

\begin{abstract}
Background: The Culicoides fauna of Algeria has been historically investigated, leading to the description of many new species by Kieffer in the 1920s, Clastrier in the 1950s or Callot in the 1960s and to a comprehensive inventory by Szadziewski in the 1980s. The emergence of bluetongue in the late 1990s enhanced Culicoides collections made in the country over the last two decades, but information remained mostly unpublished. The aim of this study is therefore to provide a comprehensive and updated checklist of Culicoides biting midge species in Algeria.

Methods: The literature (published and grey, in French and in English) from 1920 to date on Culicoides collections in Algeria was collected and analyzed in the light of the current taxonomic and systematic knowledge and methods. Fresh Culicoides material was also analyzed using light/suction trap collections carried out from November 2015 to September 2018 in nine localities of the 'wilayah' of Tiaret (northwestern Algeria). Slide mounted specimens were identified morphologically using the interactive identification key IIKC and original descriptions. Specimens were then compared with non-type material originating from different countries and partly with type material.

Results: A total of 13,709 Culicoides, belonging to at least 36 species within 10 subgenera, were examined leading to 10 new records in Algeria, including C. chiopterus, C. dewulfi, C. navaiae, C. grisescens, C. paradoxalis, C. shaklawensis, C. simulator, C. univittatus, C. achrayi and C. picturatus. These new records and all previous records provided by the literature review were discussed.

Conclusions: We propose a Culicoides checklist for the Algerian fauna of 59 valid species, including species mainly with a large Palaearctic distribution and a specific Mediterranean distribution, and only a few species from the Afrotropical region. Among them, several species, mainly of the subgenera Avaritia and Culicoides, are confirmed or probable vectors of arboviruses important in animal health.
\end{abstract}

Keywords: Ceratopogonidae, Culicoides, New records, Bluetongue, Algeria

\section{Background}

Biting midges of the genus Culicoides Latreille (Diptera: Ceratopogonidae) are small hematophagous dipterans,

\footnotetext{
*Correspondence: mimibel7425@gmail.com; thomas.balenghien@cirad.fr

2 Faculté des Sciences de la Nature et de la Vie, Université Ibn Khaldoun,

B.P.75 Zaaroura, Tiaret 1400, Algérie

${ }^{8}$ CIRAD, UMR ASTRE, 10101 Rabat, Morocco

Full list of author information is available at the end of the article
}

from 1 to $4 \mathrm{~mm}$ long [1]. Culicoides are biological vectors involved in the transmission of many arboviruses that affect humans, such as Oropouche virus, ruminants, such as bluetongue virus (BTV) and Schmallenberg virus (SBV), and equids, such as African horse sickness virus (AHSV) [2], but also several parasites, mainly nematodes that can infect humans or animals [3]. Currently, the 1368 valid species described worldwide are placed in 32 original author(s) and the source, provide a link to the Creative Commons licence, and indicate if changes were made. The images or other third party material in this article are included in the article's Creative Commons licence, unless indicated otherwise in a credit line to the material. If material is not included in the article's Creative Commons licence and your intended use is not permitted by statutory regulation or exceeds the permitted use, you will need to obtain permission directly from the copyright holder. To view a copy of this licence, visit http://creativecommons.org/licenses/by/4.0/. The Creative Commons Public Domain Dedication waiver (http://creativeco mmons.org/publicdomain/zero/1.0/) applies to the data made available in this article, unless otherwise stated in a credit line to the data. 
subgenera and 38 species groups (not placed to a subgenus), whereas $13 \%$ of the world fauna are not placed in any subgenus or group [4].

The worldwide Culicoides fauna has been diversely investigated by a number of authors. In the western Palaearctic region, several monographs and catalogues are still considered as a reference for the Culicoides fauna [5-7], whereas an interactive identification key has been developed for Culicoides females [8]. The Afrotropical fauna has benefited from various contributions, including those of Khamala \& Kettle [9] for eastern Africa and Glick [10] for Kenya in particular. Northern Africa is located in the southwestern part of the Palaearctic region and separated from the Afrotropical region by the Sahara Desert. The fauna of this region is composed mainly by species belonging to the Palaearctic fauna, along with some of the Afrotropical fauna.

In the Maghreb, Culicoides inventories have been carried out since the beginning of the 20th century, firstly with taxonomic interest enhanced in the 1960s after the emergence of AHSV in Morocco in 1965 [11-14]. In this country, additional ecological studies were carried out after the 1989-1991 AHSV outbreaks [15, 16]. Inventories of Culicoides were then re-launched in the Maghreb after the BTV emergence in the Mediterranean basin in the 2000s and the implementation of nationalscale entomological surveys $[17,18]$. Currently, 54 species of Culicoides have been recorded in Morocco [19] and 35 in Tunisia [20]. In Algeria, studies on Culicoides have been particularly intense in the first half of the 20th century, with investigations carried out during the $1920 \mathrm{~s}$ [21-23], the 1930s [24], and the 1950s [25, 26], but also after the 1965 ASHV outbreaks [27]. Altogether, these authors reported the presence of 21 species, including the following 11 still valid species described from Algeria: C. sergenti Kieffer; C. foleyi Kieffer; C. nudipennis Kieffer; C. parroti Kieffer; C. saevus Kieffer; C. sahariensis Kieffer; C. algeriensis Clastrier; C. begueti Clastrier; C. cataneii Clastrier; C. semimaculatus Clastrier; and C. marcleti Callot, Kremer \& Basset. After collections in different regions of Algeria carried out in the early 1980s, Szadziewski [28] reported 30 species, including 19 new records for the Algerian fauna, increasing the number of known species in Algeria to 40. This inventory encompassed several species known as confirmed or probable BTV vectors, such as $C$. imicola Kieffer, C. obsoletus Meigen, $C$. scoticus Downes \& Kettle within the subgenus Avaritia and C. newsteadi Austen, C. pulicaris (Linnaeus) and C. punctatus (Meigen) within the subgenus Culicoides [29-33].

From 2000 to 2011, three BTV serotypes were reported in Algeria, leading to 297 outbreaks in 2000 (BTV-2), 263 in 2006 (BTV-1) and six in 2011 (BTV-4) [34, 35]. A few years after these different episodes of BTV transmission, limited-scale surveys for Culicoides were carried out in northern and southern Algeria [36-38], before nationwide entomological surveillance was implemented in 2007 by the National Institute of Veterinary Medicine $[39,40]$. Moreover, extensive collections have been carried out in the eastern part of Algeria [41, 42]. Altogether, these recent studies increased the total number of species recorded in Algeria to 52 by identifying 12 new species for the Algerian fauna.

In this paper, we aimed to produce a comprehensive checklist of Culicoides species in Algeria, including a list of confirmed or probable BTV and AHSV vectors. For this purpose, we compiled and discussed the existing known information from published and from grey literature and examined new material collected using UV light/suction traps carried out from November 2015 to September 2018 in the 'wilayah' of Tiaret (northwestern Algeria).

\section{Methods}

Published papers (in French and English) related to Culicoides collections in Algeria were collated following searches of classical bibliographic databases (PubMed ${ }^{\circledR}$ and Google Scholar) and on institutional open access repositories (Agritrop from Cirad and Horizon from IRD). The unpublished 'grey' literature (field reports and theses) was collated from the two latter databases and from personal databases of authors, but also by contacting authors of the publications and the teams working on Culicoides in Algeria. The literature from 1920 to date was analyzed in light of the current taxonomic and systematic knowledge and methods.

We examined Culicoides individuals collected using UV light/suction traps, from November 2015 to September 2018, from nine localities in the 'wilayah' of Tiaret. The collected Culicoides were transferred to $70 \%$ ethanol for preservation. The study area is located in the western part of the 'Hauts Plateaux' (Highlands or High plateaus), a steppe-like region between the Tell and Saharan Atlas ranges in northwestern Algeria. It is dominated by a continental and semi-arid climate, characterized by very dry summers (300-500 mm per year, with a drought of 6 to 8 months) and cold winters (average annual temperature ranging from 13 to $17^{\circ} \mathrm{C}$ ) [43]. Mainly dedicated to pastoral activities, the Tiaret region encompasses forested areas composed by cork and holm oaks, Aleppo pine and cypresses.

From the material available, we selected a series of specimens that exhibited a variety of wing patterns, and also those with plain wings. Before identification, the specimens were dissected and slide mounted following the Wirth \& Martson [44] procedure. Slide mounted 
specimens were therefore identified morphologically to the species level using the interactive identification key IIKC of Mathieu et al. [8] and original descriptions. The specimens were then compared with non-type material from different countries and partly with type material available in the collection of the Institut de parasitologie et de pathologie tropicale de Strasbourg, France. To illustrate the new recordings, the wings were photographed using a Zeiss Standard 25 microscope (Zeiss, Oberkochen, Germany) equipped with a Nikon DS-Fi3 camera and Nikon NIS elements v4.6 software (Nikon corporation, Tokyo, Japan). The morphological terminology follows that of Mathieu et al. [8] and Ramilo et al. [45]. With the exception of the species composition of the subgenus Sensiculicoides, which follows Szadziewski et al. [46], the subgeneric placement of species other than the latter subgenus and distribution follow Szadziewski et al. [47].

To confirm morphological identification, a small number of specimens were sequenced for the cytochrome $c$ oxidase subunit 1 ( $\operatorname{cox} 1$ ) barcode region following the same procedure detailed in Bourquia et al. [19].

\section{Results}

A total of 13,709 Culicoides, belonging to at least 36 species within 10 subgenera, were collected in nine localities of the Tiaret region from November 2015 to September 2018. The results will be analyzed in detail in another publication. Here, we report the 10 new records from Algeria, with special emphasis on $C$. navaiae. The list of the examined material is presented in Table 1.

We report the presence of Culicoides (Avaritia) chiopterus (Meigen) (synonyms Culicoides amoenus Winnertz, 1852; Culicoides similis Goetghebuer, 1927; Culicoides dobyi Callot \& Kremer, 1969). Females may be identified using the following criteria. General aspect of the wing light greyish with little contrast as represented in Fig. 1; pale spots poorly defined and barely visible; poststigmatic pale spot covering more than one-third of the 2nd radial cell; absence of pale spots in the distal part of the wing. Hairy eyes with scattered and short interfacetal hairs, and eyes united over a short distance. First abdominal tergite with 1-2 lateral hairs. Two functional spermathecae subequal and one rudimentary. This species is distributed in the Holarctic region, including Austria, Belgium, Czech Republic, Denmark, Estonia, France with Corsica, Germany, Hungary, Ireland, Lithuania, the Near East, the Netherlands, Poland, Romania, Russia, Spain, Slovakia, Switzerland, Turkey, Ukraine [48], the UK and in the Nearctic region. To date, only two species with hairy eyes have been recorded in the Palaearctic region, of which C. chiopterus is the only one described from North Africa. We produced a cox 1 barcode sequence of the C. chiopterus female (GenBank: MT782149) to confirm the morphological identification.

We report the presence of Culicoides (Avaritia) dewulfi Goetghebuer (synonym Culicoides pseudochiopterus Downes \& Kettle, 1952). Females may be identified using the following criteria. General aspect of the wing greyish and well contrasted with the darker part between the 1st and the 2nd radial cells as represented in Fig. 2; pale spots poorly defined; poststigmatic pale spot covering more than one third of the 2nd radial cell; the distal pale spot/ area of $r 3$ larger than the pale spot/area in the distal part of $\mathrm{m} 1$. Eyes bare and joined over a short distance. First abdominal tergite with 8-12 lateral hairs. Two functional spermathecae unequal and one rudimentary. This Palaearctic species is widely distributed in Europe, including Belgium, Czech Republic, Denmark, Estonia, France with Corsica, Germany, Italy, Poland, Romania, Russia, Slovakia, Spain, Switzerland and the UK [48]. While phylogenetically well separated from species of the Obsoletus group [49], the wing pattern of C. dewulfi shares similarities with the latter group. By prior confirmation of slide mounted specimens, the combination of (i) the contrasted wing pattern, (ii) the pale spot/area in the distal part of $\mathrm{r} 3$ larger than that of $\mathrm{m} 1$ and (iii) the numerous lateral hairs on the first abdominal tergite are good indicators of the species under stereomicroscopy.

We report the presence of Culicoides (Beltranmyia) navaiae Lane. Females may be identified using the following criteria. Wing with a few very faint spots as represented in Fig. 3; no spots in the distal part of r3; absence of macrotrichia in the basal cell. Eyes bare and closely separated (Fig. 4). Sensilla coeloconica present on flagellomeres 1, 9-12. Absence of a postpharyngal armature. Third palpal segment triangular and moderately swollen with single, wide and shallow sensory pit. One functional spermathecae without a pigmented neck (Fig. 5). Males may be identified using the following criteria. Wings pale with fainter spots than in females and barely visible. Ninth tergite broad with a median deep notch, short and divergent apicolateral processes; ninth sternite with bare ventral membrane. Aedeagus with rounded basal arch extending more than two-thirds of the total length, slender and curved-ended basal arms, distal process moderately long (one third of the total length) and bluntended (Fig. 6). Parameres separated, swollen at the base and tapering distally to a slender point. This species is reported in the Arabian Peninsula especially in Bahrain, Saudi Arabia, and in the Sinai [50-52]. Culicoides navaiae is the only Beltranmyia species from the Palaearctic and the Afrotropical regions exhibiting the combination of the following characters: very faint wing pattern close to the plain wing aspect, no spots on the distal part of $r 3$ and sensilla coeloconia distribution on flagellomeres 1 , 
Table 1 List of the material examined to produce 10 new records for the Algerian fauna

\begin{tabular}{|c|c|c|c|c|c|c|}
\hline Species & Type of material & Country & Locality & Coordinates & Date & Material examined \\
\hline \multirow{7}{*}{$\begin{array}{l}\text { Culicoides (Avaritia) chiop- } \\
\text { terus (Meigen) }\end{array}$} & \multirow[t]{7}{*}{ Non-type material } & Algeria & Ain El Deheb & $34^{\circ} 51^{\prime} \mathrm{N}, 1^{\circ} 29^{\prime} \mathrm{E}$ & 23-24 Mar 2018 & 1 female \\
\hline & & \multirow[t]{6}{*}{ France } & Andevanne & $49^{\circ} 23^{\prime} \mathrm{N}, 5^{\circ} 4^{\prime} \mathrm{E}$ & 17-18 Oct 2006 & 1 female \\
\hline & & & Caro & $43^{\circ} 8^{\prime} \mathrm{N}, 1^{\circ} 14^{\prime} \mathrm{W}$ & 23-24 Oct 2006 & 1 female \\
\hline & & & Nuillé sur Vicoin & $47^{\circ} 57^{\prime} \mathrm{N}, 0^{\circ} 45^{\prime} \mathrm{W}$ & 4-5 Jul 2011 & 1 female \\
\hline & & & Orbey & $48^{\circ} 7^{\prime} \mathrm{N}, 7^{\circ} 7^{\prime} \mathrm{E}$ & 25-26 May 2009 & 5 females \\
\hline & & & Pontivy & $48^{\circ} 4^{\prime} \mathrm{N}, 2^{\circ} 58^{\prime} \mathrm{W}$ & 18-19 Jan 2012 & 5 females \\
\hline & & & Schwobsheim & $48^{\circ} 13^{\prime} \mathrm{N}, 7^{\circ} 34^{\prime} \mathrm{E}$ & 18-19 Apr 2011 & 1 female \\
\hline \multirow{11}{*}{$\begin{array}{l}\text { Culicoides (Avaritia) dewulfi } \\
\text { Goetghebuer }\end{array}$} & \multirow[t]{11}{*}{ Non-type material } & \multirow[t]{8}{*}{ Algeria } & Tiaret & $35^{\circ} 23^{\prime} \mathrm{N}, 1^{\circ} 21^{\prime} \mathrm{E}$ & 16-19 Sep 2017 & 1 female \\
\hline & & & Bougara & $35^{\circ} 29^{\prime} \mathrm{N}, 1^{\circ} 55^{\prime} \mathrm{E}$ & 13-15 Nov 2016 & 1 female \\
\hline & & & Sougueur & $35^{\circ} 09^{\prime} \mathrm{N}, 1^{\circ} 31^{\prime} \mathrm{E}$ & 13-16 May 2016 & 2 females \\
\hline & & & Ain El Deheb & $34^{\circ} 51^{\prime} \mathrm{N}, 1^{\circ} 29^{\prime} \mathrm{E}$ & 25-27 Jul 2017 & 3 females \\
\hline & & & Ain El Deheb & $34^{\circ} 51^{\prime} \mathrm{N}, 1^{\circ} 29^{\prime} \mathrm{E}$ & 1-2 Jun 2018 & 1 female \\
\hline & & & Hammadia & $35^{\circ} 26^{\prime} \mathrm{N}, 1^{\circ} 53^{\prime} \mathrm{E}$ & 2-5 Jun 2016 & 1 female \\
\hline & & & Takhmaret & $35^{\circ} 06^{\prime} \mathrm{N}, 0^{\circ} 42^{\prime} \mathrm{E}$ & 12-15 Sep 2017 & 1 female \\
\hline & & & Takhmaret & $35^{\circ} 06^{\prime} \mathrm{N}, 0^{\circ} 42^{\prime} \mathrm{E}$ & 13-14 Sep 2018 & 3 females \\
\hline & & \multirow[t]{3}{*}{ France } & Caro & $43^{\circ} 84 \mathrm{~N}, 1^{\circ} 14^{\prime} \mathrm{W}$ & 29-30 May 2006 & 2 females \\
\hline & & & Cuguen & $48^{\circ} 26^{\prime} \mathrm{N}, 1^{\circ} 39^{\prime} \mathrm{W}$ & 14-15 Nov 2011 & 1 female \\
\hline & & & Lazer & $44^{\circ} 21^{\prime} \mathrm{N}, 5^{\circ} 51^{\prime} \mathrm{E}$ & 18-19 Apr 2011 & 2 females \\
\hline \multirow{30}{*}{$\begin{array}{l}\text { Culicoides (Beltranmyia) } \\
\text { navaiae Lane }\end{array}$} & \multirow[t]{30}{*}{ Non-type material } & \multirow[t]{28}{*}{ Algeria } & Tiaret & $35^{\circ} 23^{\prime} \mathrm{N}, 1^{\circ} 21^{\prime} \mathrm{E}$ & 3-6 Jun 2017 & 2 females \\
\hline & & & Tiaret & $35^{\circ} 23^{\prime} \mathrm{N}, 1^{\circ} 21^{\prime} \mathrm{E}$ & 28-30 Sep 2017 & 1 male, 2 females \\
\hline & & & Tiaret & $35^{\circ} 23^{\prime} \mathrm{N}, 1^{\circ} 21^{\prime} \mathrm{E}$ & 21-22 Sep 2018 & 2 females \\
\hline & & & Bougara & $35^{\circ} 29^{\prime} \mathrm{N}, 1^{\circ} 55^{\prime} \mathrm{E}$ & 9-12 May 2016 & 3 females \\
\hline & & & Bougara & $35^{\circ} 29^{\prime} \mathrm{N}, 1^{\circ} 55^{\prime} \mathrm{E}$ & 10-13 Nov 2016 & 3 females \\
\hline & & & Bougara & $35^{\circ} 29^{\prime} \mathrm{N}, 1^{\circ} 55^{\prime} \mathrm{E}$ & 15-19 Apr 2017 & 5 females \\
\hline & & & Bougara & $35^{\circ} 29^{\prime} \mathrm{N}, 1^{\circ} 55^{\prime} \mathrm{E}$ & 29-30 Jun 2018 & 1 female \\
\hline & & & Sougueur & $35^{\circ} 09^{\prime} \mathrm{N}, 1^{\circ} 31^{\prime} \mathrm{E}$ & 3-6 Feb 2016 & 11 females \\
\hline & & & Sougueur & $35^{\circ} 09^{\prime} \mathrm{N}, 1^{\circ} 31^{\prime} \mathrm{E}$ & 23-26 Jun 2017 & 4 females \\
\hline & & & Sougueur & $35^{\circ} 09^{\prime} \mathrm{N}, 1^{\circ} 31^{\prime} \mathrm{E}$ & 14-15 Sep 2018 & 2 females \\
\hline & & & Ain El Deheb & $34^{\circ} 51^{\prime} \mathrm{N}, 1^{\circ} 29^{\prime} \mathrm{E}$ & 2-5 Apr 2016 & 11 females \\
\hline & & & Ain El Deheb & $34^{\circ} 51^{\prime} \mathrm{N}, 1^{\circ} 29^{\prime} \mathrm{E}$ & 24-27 Apr 2017 & 2 females \\
\hline & & & Ain El Deheb & $34^{\circ} 51^{\prime} \mathrm{N}, 1^{\circ} 29^{\prime} \mathrm{E}$ & 21-22 Sep 2018 & 27 females \\
\hline & & & Ksar Chellala & $35^{\circ} 15^{\prime} \mathrm{N}, 2^{\circ} 18^{\prime} \mathrm{E}$ & 13-16 May 2016 & 20 females \\
\hline & & & Ksar Chellala & $35^{\circ} 15^{\prime} \mathrm{N}, 2^{\circ} 18^{\prime} \mathrm{E}$ & 1-4 Nov 2017 & 18 females \\
\hline & & & Ksar Chellala & $35^{\circ} 15^{\prime} \mathrm{N}, 2^{\circ} 18^{\prime} \mathrm{E}$ & 21-22 Sep 2018 & 18 females \\
\hline & & & Hammadia & $35^{\circ} 26^{\prime} \mathrm{N}, 1^{\circ} 53^{\prime} \mathrm{E}$ & 16-19 Jun 2016 & 12 females \\
\hline & & & Hammadia & $35^{\circ} 26^{\prime} \mathrm{N}, 1^{\circ} 53^{\prime} \mathrm{E}$ & 1-4 Jul 2017 & 18 females \\
\hline & & & Hammadia & $35^{\circ} 26^{\prime} \mathrm{N}, 1^{\circ} 53^{\prime} \mathrm{E}$ & 7-8 Sep 2018 & 9 females \\
\hline & & & Rahouia & $35^{\circ} 29^{\prime} \mathrm{N}, 1^{\circ} 03^{\prime} \mathrm{E}$ & 23-26 Jun 2017 & 22 females \\
\hline & & & Rahouia & $35^{\circ} 29^{\prime} \mathrm{N}, 1^{\circ} 03^{\prime} \mathrm{E}$ & 9-12 Jul 2017 & 11 females \\
\hline & & & Rahouia & $35^{\circ} 29^{\prime} \mathrm{N}, 1^{\circ} 03^{\prime} \mathrm{E}$ & $21-22 \operatorname{Sep} 2018$ & 18 females \\
\hline & & & Machraa S'fa & $35^{\circ} 22^{\prime} \mathrm{N}, 1^{\circ} 03^{\prime} \mathrm{E}$ & 3-6 Jun 2017 & 12 females \\
\hline & & & Machraa S'fa & $35^{\circ} 22^{\prime} \mathrm{N}, 1^{\circ} 03^{\prime} \mathrm{E}$ & 23-26 Jun 2017 & 23 females \\
\hline & & & Machraa S'fa & $35^{\circ} 22^{\prime} \mathrm{N}, 1^{\circ} 03^{\prime} \mathrm{E}$ & 13-14 Jul 2018 & 7 females \\
\hline & & & Takhmaret & $35^{\circ} 06^{\prime} \mathrm{N}, 0^{\circ} 42^{\prime} \mathrm{E}$ & 5-8 Jul 2016 & 8 females \\
\hline & & & Takhmaret & $35^{\circ} 06^{\prime} \mathrm{N}, 0^{\circ} 42^{\prime} \mathrm{E}$ & 29 Jul-1 Aug 2017 & 6 females \\
\hline & & & Takhmaret & $35^{\circ} 06^{\prime} \mathrm{N}, 0^{\circ} 42^{\prime} \mathrm{E}$ & 8-9 Jun 2018 & 9 females \\
\hline & & \multirow[t]{2}{*}{ Egypt } & Sinaï, Khirba & $31^{\circ} 1^{\prime} \mathrm{N}, 32^{\circ} 53^{\prime} \mathrm{E}$ & 25 Apr 1979 & 10 females \\
\hline & & & Sinaï, Khirba & $31^{\circ} 1^{\prime} \mathrm{N}, 32^{\circ} 53^{\prime} \mathrm{E}$ & 24 Apr 1979 & 1 male \\
\hline
\end{tabular}


Table 1 (continued)

\begin{tabular}{|c|c|c|c|c|c|c|}
\hline Species & Type of material & Country & Locality & Coordinates & Date & Material examined \\
\hline \multirow{31}{*}{$\begin{array}{l}\text { Culicoides (Culicoides) grises- } \\
\text { cens Edwards }\end{array}$} & \multirow[t]{31}{*}{ Non-type material } & \multirow[t]{29}{*}{ Algeria } & Sougueur & $35^{\circ} 09^{\prime} \mathrm{N}, 1^{\circ} 31^{\prime} \mathrm{E}$ & 5-7 Apr 2017 & 10 females \\
\hline & & & Sougueur & $35^{\circ} 09^{\prime} \mathrm{N}, 1^{\circ} 31^{\prime} \mathrm{E}$ & 26-29 May 2017 & 13 females \\
\hline & & & Sougueur & $35^{\circ} 09^{\prime} \mathrm{N}, 1^{\circ} 31^{\prime} \mathrm{E}$ & 3-6 Jun 2017 & 5 females \\
\hline & & & Sougueur & $35^{\circ} 09^{\prime} \mathrm{N}, 1^{\circ} 31^{\prime} \mathrm{E}$ & 17-20 Jul 2017 & 13 females \\
\hline & & & Sougueur & $35^{\circ} 09^{\prime} \mathrm{N}, 1^{\circ} 31^{\prime} \mathrm{E}$ & 25-28 Jul 2017 & 11 females \\
\hline & & & Sougueur & $35^{\circ} 09^{\prime} \mathrm{N}, 1^{\circ} 31^{\prime} \mathrm{E}$ & 20-23 Sep 2017 & 15 females \\
\hline & & & Sougueur & $35^{\circ} 09^{\prime} \mathrm{N}, 1^{\circ} 31^{\prime} \mathrm{E}$ & 1-3 Oct 2017 & 12 females \\
\hline & & & Sougueur & $35^{\circ} 09^{\prime} \mathrm{N}, 1^{\circ} 31^{\prime} \mathrm{E}$ & 1-4 Jul 2018 & 9 females \\
\hline & & & Sougueur & $35^{\circ} 09^{\prime} \mathrm{N}, 1^{\circ} 31^{\prime} \mathrm{E}$ & 7-8 Sep 2018 & 9 females \\
\hline & & & Ain El Deheb & $34^{\circ} 51^{\prime} \mathrm{N}, 1^{\circ} 29^{\prime} \mathrm{E}$ & 29 Nov -5 Dec 2015 & 2 females \\
\hline & & & Ain El Deheb & $34^{\circ} 51^{\prime} \mathrm{N}, 1^{\circ} 29^{\prime} \mathrm{E}$ & 15-17 Sep 2016 & 15 females \\
\hline & & & Ain El Deheb & $34^{\circ} 51^{\prime} \mathrm{N}, 1^{\circ} 29^{\prime} \mathrm{E}$ & 5-7 Apr 2017 & 9 females \\
\hline & & & Ain El Deheb & $34^{\circ} 51^{\prime} \mathrm{N}, 1^{\circ} 29^{\prime} \mathrm{E}$ & 28-29 Sep 2018 & 11 females \\
\hline & & & Ksar Chellala & $35^{\circ} 15^{\prime} \mathrm{N}, 2^{\circ} 18^{\prime} \mathrm{E}$ & 26-29 May 2017 & 7 females \\
\hline & & & Ksar Chellala & $35^{\circ} 15^{\prime} \mathrm{N}, 2^{\circ} 18^{\prime} \mathrm{E}$ & 9-12 Nov 2017 & 6 females \\
\hline & & & Ksar Chellala & $35^{\circ} 15^{\prime} \mathrm{N}, 2^{\circ} 18^{\prime} \mathrm{E}$ & 21-22 Sep 2018 & 16 females \\
\hline & & & Hammadia & $35^{\circ} 26^{\prime} \mathrm{N}, 1^{\circ} 53^{\prime} \mathrm{E}$ & 15-17 Sep 2016 & 8 females \\
\hline & & & Hammadia & $35^{\circ} 26^{\prime} \mathrm{N}, 1^{\circ} 53^{\prime} \mathrm{E}$ & 28-30 Sep 2017 & 7 females \\
\hline & & & Hammadia & $35^{\circ} 26^{\prime} \mathrm{N}, 1^{\circ} 53^{\prime} \mathrm{E}$ & 1-3 Oct 2017 & 11 females \\
\hline & & & Hammadia & $35^{\circ} 26^{\prime} \mathrm{N}, 1^{\circ} 53^{\prime} \mathrm{E}$ & 28-29 Sep 2018 & 12 females \\
\hline & & & Rahouia & $35^{\circ} 29^{\prime} \mathrm{N}, 1^{\circ} 03^{\prime} \mathrm{E}$ & 24-27 Jun 2016 & 5 females \\
\hline & & & Rahouia & $35^{\circ} 29^{\prime} \mathrm{N}, 1^{\circ} 03^{\prime} \mathrm{E}$ & 12-15 Sep 2017 & 8 females \\
\hline & & & Rahouia & $35^{\circ} 29^{\prime} \mathrm{N}, 1^{\circ} 03^{\prime} \mathrm{E}$ & 21-22 Sep 2018 & 7 females \\
\hline & & & Machraa S'fa & $35^{\circ} 22^{\prime} \mathrm{N}, 1^{\circ} 03^{\prime} \mathrm{E}$ & 8-10 Sep 2016 & 9 females \\
\hline & & & Machraa S'fa & $35^{\circ} 22^{\prime} \mathrm{N}, 1^{\circ} 03^{\prime} \mathrm{E}$ & 26-29 May 2017 & 9 females \\
\hline & & & Machraa S'fa & $35^{\circ} 22^{\prime} \mathrm{N}, 1^{\circ} 03^{\prime} \mathrm{E}$ & 15-16 Jun 2018 & 7 females \\
\hline & & & Takhmaret & $35^{\circ} 06^{\prime} \mathrm{N}, 0^{\circ} 42^{\prime} \mathrm{E}$ & 20-23 Jun 2016 & 19 females \\
\hline & & & Takhmaret & $35^{\circ} 06^{\prime} \mathrm{N}, 0^{\circ} 42^{\prime} \mathrm{E}$ & 5-7 Apr 2017 & 10 females \\
\hline & & & Takhmaret & $35^{\circ} 06^{\prime} \mathrm{N}, 0^{\circ} 42^{\prime} \mathrm{E}$ & 28-29 Sep 2018 & 12 females \\
\hline & & \multirow[t]{2}{*}{ France } & Brognon & $49^{\circ} 55^{\prime} \mathrm{N}, 4^{\circ} 18^{\prime} \mathrm{E}$ & 2è-28 Jun 2007 & 1 female \\
\hline & & & Marcoux & $44^{\circ} 7^{\prime} \mathrm{N}, 6^{\circ} 17^{\prime} \mathrm{E}$ & 22-23 Jul 2009 & 4 females \\
\hline \multirow{9}{*}{$\begin{array}{l}\text { Culicoides (Culicoides) para- } \\
\text { doxalis Ramilo \& Delécolle }\end{array}$} & \multirow[t]{9}{*}{ Type material } & \multirow{9}{*}{$\begin{array}{l}\text { Algeria, } \\
\text { France, Corsica }\end{array}$} & Ain El Deheb & $34^{\circ} 51^{\prime} \mathrm{N}, 1^{\circ} 29^{\prime} \mathrm{E}$ & 27-28 Apr 2018 & 1 female \\
\hline & & & Pietra Corbara & $42^{\circ} 50^{\prime} \mathrm{N}, 9^{\circ} 26^{\prime} \mathrm{E}$ & 3-4 Jun 2003 & 1 female (holotype) \\
\hline & & & Pietra Corbara & $42^{\circ} 50^{\prime} \mathrm{N}, 9^{\circ} 26^{\prime} \mathrm{E}$ & 3-4 Jun 2003 & 2 female \\
\hline & & & Pietra Corbara & $42^{\circ} 50^{\prime} \mathrm{N}, 9^{\circ} 26^{\prime} \mathrm{E}$ & 6-7 Jul 2004 & 1 female \\
\hline & & & Porto Vecchio & $41^{\circ} 35^{\prime} \mathrm{N}, 9^{\circ} 15^{\prime} \mathrm{E}$ & 22-23 Sep 2005 & 2 females \\
\hline & & & Sarrola Carcopino & $42^{\circ} 0^{\prime} \mathrm{N}, 8^{\circ} 50^{\prime} \mathrm{E}$ & 26-27 Jun 2006 & 1 female \\
\hline & & & Sartene & $41^{\circ} 38^{\prime} \mathrm{N}, 8^{\circ} 57^{\prime} \mathrm{E}$ & 27-28 Sep 2005 & 1 female \\
\hline & & & Sartene & $41^{\circ} 38^{\prime} \mathrm{N}, 8^{\circ} 57^{\prime} \mathrm{E}$ & 12-13 Jun 2002 & 1 female \\
\hline & & & Sartene & $41^{\circ} 38^{\prime} \mathrm{N}, 8^{\circ} 57^{\prime} \mathrm{E}$ & 20-21 Jun 2002 & 1 female \\
\hline
\end{tabular}


Table 1 (continued)

\begin{tabular}{|c|c|c|c|c|c|c|}
\hline Species & Type of material & Country & Locality & Coordinates & Date & Material examined \\
\hline \multirow{7}{*}{$\begin{array}{l}\text { Culicoides (Sensiculicoides) } \\
\text { shaklawensis Khalaf }\end{array}$} & \multirow[t]{7}{*}{ Non-type material } & \multirow[t]{4}{*}{ Algeria } & Tiaret & $35^{\circ} 23^{\prime} \mathrm{N}, 1^{\circ} 21^{\prime} \mathrm{E}$ & 9-14 Mar 2016 & 1 female \\
\hline & & & Tiaret & $35^{\circ} 23^{\prime} \mathrm{N}, 1^{\circ} 21^{\prime} \mathrm{E}$ & 15-16 Jun 2018 & 1 female \\
\hline & & & Tiaret & $35^{\circ} 23^{\prime} \mathrm{N}, 1^{\circ} 21^{\prime} \mathrm{E}$ & 27-28 Jul 2018 & 1 female \\
\hline & & & Bougara & $35^{\circ} 29^{\prime} \mathrm{N}, 1^{\circ} 55^{\prime} \mathrm{E}$ & 29-31 Oct 2016 & 1 female \\
\hline & & \multirow[t]{3}{*}{ France } & Calvi & $42^{\circ} 32^{\prime} \mathrm{N}, 8^{\circ} 45^{\prime} \mathrm{E}$ & 23-24 Jun 2003 & 1 female \\
\hline & & & Marcoux & $44^{\circ} 7^{\prime} \mathrm{N}, 6^{\circ} 17^{\prime} \mathrm{E}$ & 22-23 Jul 2009 & 1 female \\
\hline & & & Moltifao & $42^{\circ} 28^{\prime} \mathrm{N}, 9^{\circ} 7^{\prime} \mathrm{E}$ & 5-6 Aug 2003 & 1 female \\
\hline \multirow{5}{*}{$\begin{array}{l}\text { Culicoides (Sensiculicoides) } \\
\text { simulator Edwards }\end{array}$} & \multirow[t]{5}{*}{ Non-type material } & Algeria & Tiaret & $35^{\circ} 23^{\prime} \mathrm{N}, 1^{\circ} 21^{\prime} \mathrm{E}$ & 1-2 Jun 2018 & 1 female \\
\hline & & \multirow[t]{4}{*}{ France } & Aleria & $42^{\circ} 6^{\prime} \mathrm{N}, 9^{\circ} 29^{\prime} \mathrm{E}$ & 8-9 Jun 2004 & 1 female \\
\hline & & & La Chapelle d'Andaine & $48^{\circ} 31^{\prime} \mathrm{N}, 0^{\circ} 28^{\prime} \mathrm{W}$ & 19-20 Jun 2012 & 1 female \\
\hline & & & Marcoux & $44^{\circ} 7^{\prime} \mathrm{N}, 6^{\circ} 17^{\prime} \mathrm{E}$ & 22-23 Jul 2009 & 1 female \\
\hline & & & Remilly Aillicourt & $49^{\circ} 39^{\prime} \mathrm{N}, 4^{\circ} 58^{\prime} \mathrm{E}$ & 24-25 May 2007 & 1 female \\
\hline \multirow{8}{*}{$\begin{array}{l}\text { Culicoides (Sensiculicoides) } \\
\text { univittatus Vimmer }\end{array}$} & \multirow[t]{8}{*}{ Non-type material } & \multirow[t]{3}{*}{ Algeria } & Tiaret & $35^{\circ} 23^{\prime} \mathrm{N}, 1^{\circ} 21^{\prime} \mathrm{E}$ & 10-12 Dec 2016 & 1 male, 1 female \\
\hline & & & Tiaret & $35^{\circ} 23^{\prime} \mathrm{N}, 1^{\circ} 21^{\prime} \mathrm{E}$ & 15-17 Mar 2017 & 4 males, 7 females \\
\hline & & & Tiaret & $35^{\circ} 23^{\prime} \mathrm{N}, 1^{\circ} 21^{\prime} \mathrm{E}$ & 4-5 May 2018 & 2 females \\
\hline & & \multirow[t]{5}{*}{ France, Corsica } & Figari & $41^{\circ} 30^{\prime} \mathrm{N}, 9^{\circ} 5^{\prime} \mathrm{E}$ & 10-11 Apr 2006 & 1 male, 4 females \\
\hline & & & Figari & $41^{\circ} 30^{\prime} \mathrm{N}, 9^{\circ} 5^{\prime} \mathrm{E}$ & 14-15 Feb 2011 & 1 female \\
\hline & & & Moltifao & $42^{\circ} 28^{\prime} \mathrm{N}, 9^{\circ} 7^{\prime} \mathrm{E}$ & 25-26 Apr 2005 & 1 female \\
\hline & & & San Giuliano & $42^{\circ} 17^{\prime} \mathrm{N}, 9^{\circ} 32^{\prime} \mathrm{E}$ & 6-7 Mar 2003 & 2 males \\
\hline & & & San Giuliano & $42^{\circ} 17^{\prime} \mathrm{N}, 9^{\circ} 32^{\prime} \mathrm{E}$ & 10-11 Feb 2011 & 1 female \\
\hline \multirow{5}{*}{$\begin{array}{l}\text { Culicoides (Silvaticulicoides) } \\
\text { achrayi Kettle \& Lawson }\end{array}$} & \multirow[t]{5}{*}{ Non-type material } & \multirow[t]{3}{*}{ Algeria } & Hammadia & $35^{\circ} 26^{\prime} \mathrm{N}, 1^{\circ} 53^{\prime} \mathrm{E}$ & 27-28 Apr 2018 & 1 female \\
\hline & & & Sougueur & $35^{\circ} 09^{\prime} \mathrm{N}, 1^{\circ} 31^{\prime} \mathrm{E}$ & 15-17 Oct 2016 & 1 female \\
\hline & & & Sougueur & $35^{\circ} 09^{\prime} \mathrm{N}, 1^{\circ} 31^{\prime} \mathrm{E}$ & 5-7 Apr 2017 & 1 female \\
\hline & & \multirow[t]{2}{*}{ France } & Crozon & $48^{\circ} 16^{\prime} \mathrm{N}, 4^{\circ} 30^{\prime} \mathrm{W}$ & 13-14 Jun 2011 & 2 females \\
\hline & & & Pontivy & $48^{\circ} 4^{\prime} \mathrm{N}, 2^{\circ} 58^{\prime} \mathrm{W}$ & 18-19 Jan 2012 & 3 females \\
\hline \multirow{7}{*}{$\begin{array}{l}\text { Culicoides (Silvaticulicoides) } \\
\text { picturatus Kremer \& Deduit }\end{array}$} & \multirow[t]{7}{*}{ Non-type material } & \multirow[t]{3}{*}{ Algeria } & Tiaret & $35^{\circ} 23^{\prime} \mathrm{N}, 1^{\circ} 21^{\prime} \mathrm{E}$ & 24-27 Jun 2016 & 1 female, 1 male \\
\hline & & & Tiaret & $35^{\circ} 23^{\prime} \mathrm{N}, 1^{\circ} 21^{\prime} \mathrm{E}$ & 10-12 Jul 2016 & 1 female \\
\hline & & & Tiaret & $35^{\circ} 23^{\prime} \mathrm{N}, 1^{\circ} 21^{\prime} \mathrm{E}$ & 28-29 Sep 2018 & 1 female \\
\hline & & \multirow[t]{4}{*}{ France } & Humes Jorquenay & $47^{\circ} 54^{\prime} \mathrm{N}, 5^{\circ} 18^{\prime} \mathrm{E}$ & 6-7 Jun 2011 & 1 male \\
\hline & & & Lazer & $44^{\circ} 21^{\prime} \mathrm{N}, 5^{\circ} 51^{\prime} \mathrm{E}$ & 18-19 Apr 2011 & 1 female \\
\hline & & & Porto Vecchio & $41^{\circ} 35^{\prime} \mathrm{N}, 9^{\circ} 15^{\prime} \mathrm{E}$ & 29-30 May 2002 & 2 males \\
\hline & & & Saignon & $43^{\circ} 51^{\prime} \mathrm{N}, 5^{\circ} 27^{\prime} \mathrm{E}$ & 6-7 Jun 2011 & 3 females \\
\hline
\end{tabular}
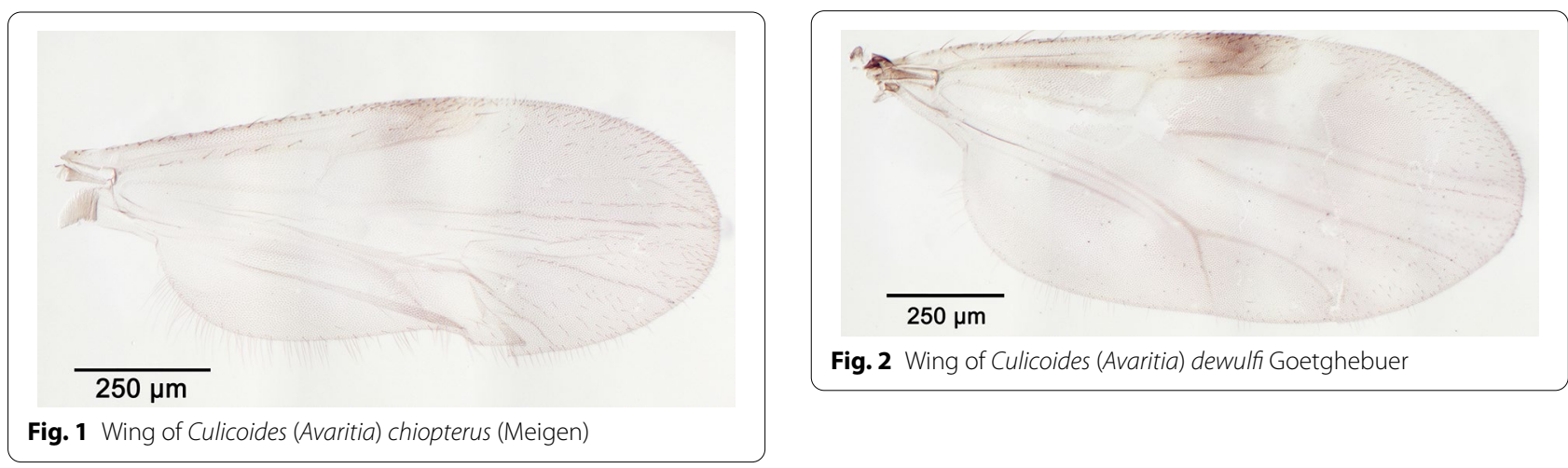
9-12. The wing pattern of C. navaiae may appear similar to the unspotted wing of $C$. homochrous Remm, but the latter has a sensilla coeloconica distribution on flagellomeres 1-2, 7, 9, 11-12 [53]. However, Glukhova [54] reported for $C$. homochrous the sensilla distribution on flagellomeres 1-3, (7), 9-12 and thus the morphological characteristics of this species should be clarified.

We report the presence of Culicoides (Culicoides) grisescens Edwards (synonyms Culicoides remmi DamianGeorgescu, 1972; Culicoides arschanicus Mirzaeva, 1984). Females may be identified using the following criteria. Spotted wing as represented in Fig. 7; cual cell pale. Eyes bare, joined over a short distance or separated but connected by a suture. Third palpal segment slender or slightly swollen with multiple and irregular pits. Two functional spermathecae subequal; short pigmented neck and one rudimentary spermathecae. Foreleg and hind leg with spines on first and second tarsomeres; middle leg with spines from first to fourth tarsomeres. This species is distributed in the northern and eastern parts of the Palaearctic region, including Belgium, Denmark [55], Estonia, France, Germany, Poland, Romania, Russia, Slovakia, Switzerland and Ukraine [48]. A phylogenetic study based on ITS2 markers and morphological observations led their authors to suggest that C. remmi Damian-Georgescu should be raised from synonymy with $C$. grisescens $[56,57]$. We produced a cox 1 barcode sequence of a C. grisescens female (GenBank: MT782148) to confirm the morphological identification.

We report the presence of Culicoides (Culicoides) paradoxalis Ramilo \& Delécolle. Females may be identified using the following criteria. Spotted wing as represented in Fig. 8; pale spot in the proximal part of $\mathrm{m} 2$ smaller than the one in $\mathrm{m} 1$ or absent; poststigmatic pale spot covering half of the 2nd radial cell; cual cell centered by a dark spot. Eyes bare and joined over a short distance. Two functional spermathecae subequal and one rudimentary. Middle leg with spines on tarsal segments 1 to 3. This species is distributed in France and Portugal [45]. In recent years, the morphological and molecular diversities within the subgenus Culicoides have been studied, leading to the description of several new species [58-60].

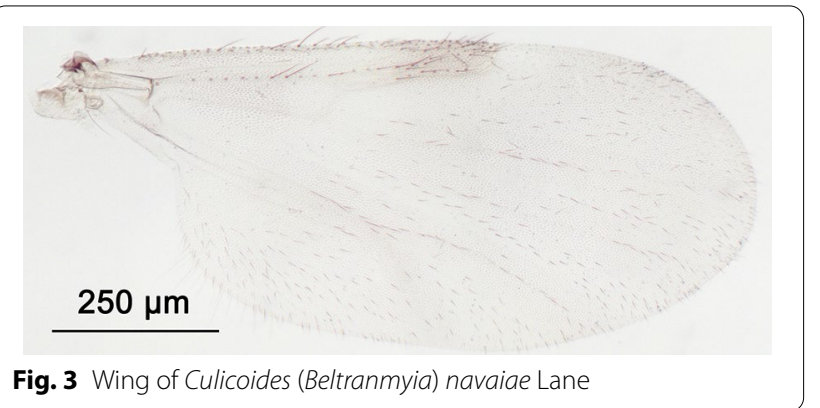

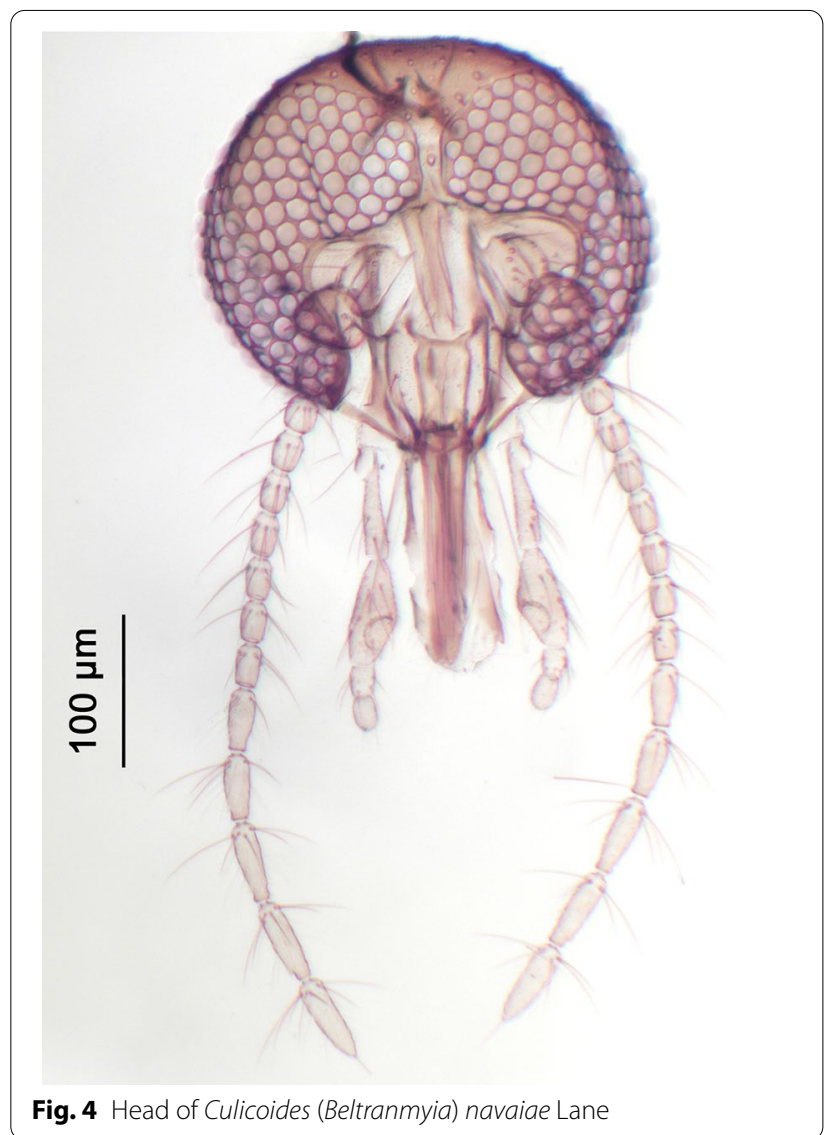

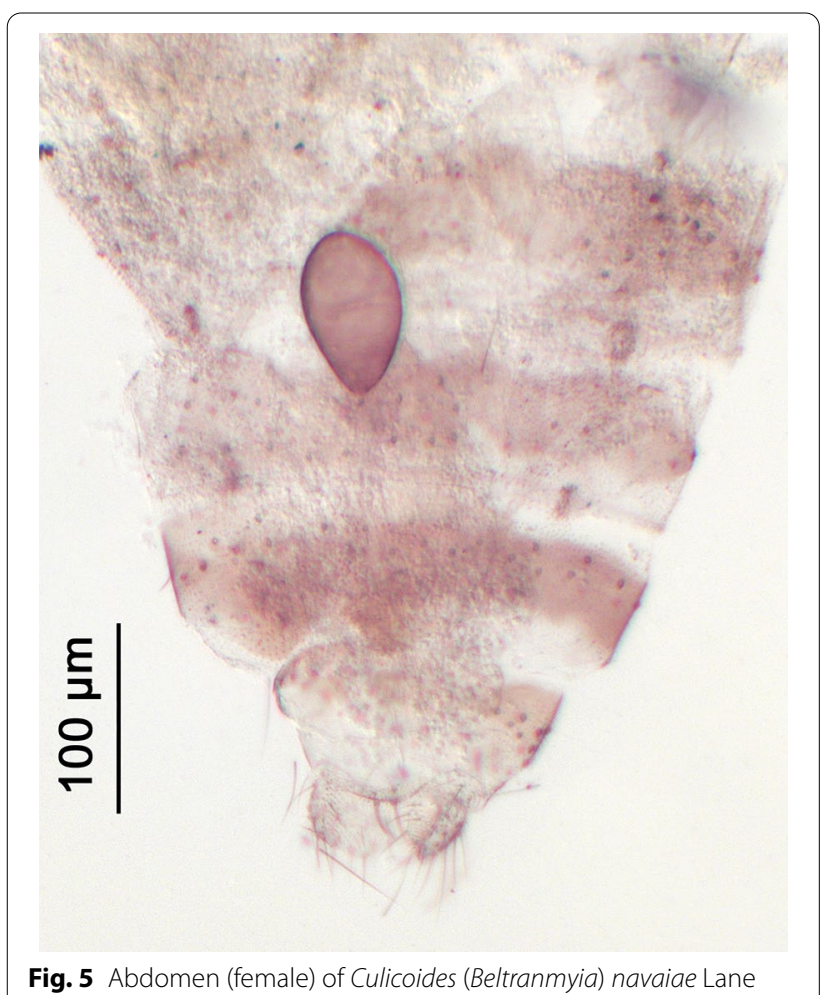




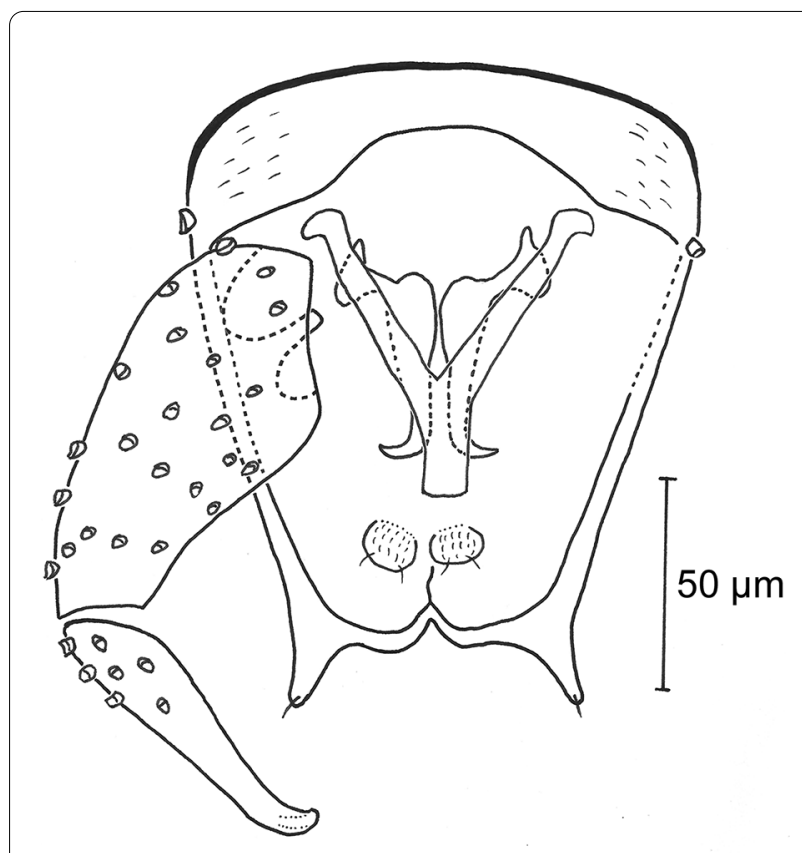

Fig. 6 Male genitalia of Culicoides (Beltranmyia) navaiae Lane

Nevertheless, observation of the wing pattern, the pale spot in cell $\mathrm{m} 2$ in particular, and the absence of spines on the fourth mid tarsomere should allow the identification of C. paradoxalis with confidence.

We report the presence of Culicoides (Sensiculicoides) shaklawensis Khalaf (synonym Culicoides caspius Gutsevich, 1959). Females may be identified using the following criteria. Spotted wing as represented in Fig. 9; second radial cell completely dark with the $\mathrm{R} 3$ vein slightly covered by the poststigmatic pale spot; most of the veins M1, M2 and CuA1 pale with the exception of the distal tip dark. Eyes bare and separated narrowly. Sensilla coeloconica present on flagellomeres 1, 9-13. Two functional spermathecae unequal and one rudimentary. Middle leg with spines on tarsal segments 1 to 3 . Described from Iraq, this species is widespread in the Middle East [61], and reported from Bulgaria, Cyprus [61], Czech Republic, France with Corsica [62], Italy, Morocco, Slovakia,

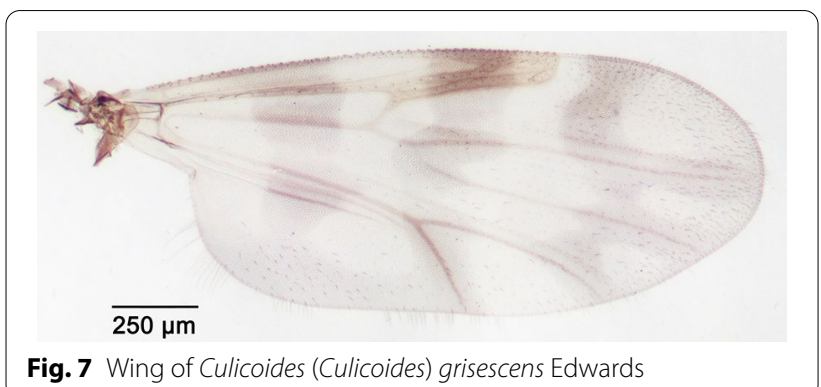

Fig. 7 Wing of Culicoides (Culicoides) grisescens Edwards
Spain, Tunisia, Turkey and Ukraine. The very characteristic wing pattern of $C$. shaklawensis can be used to identify this species with certainty in the Palaearctic region. In Central Asia, C. kugitangi Ataev described in Turkmenistan has a wing pattern similar to that of $C$. shaklawanesis and females can be easily separated from the latter species by the sensilla coeloconica distribution present on all flagellomeres [54].

We report the presence of Culicoides (Sensiculicoides) simulator Edwards. Females may be identified using the following criteria. Spotted wing as represented in Fig. 10; poststigmatic pale spot covering one third of the 2 nd radial cell; pale spot on the $\mathrm{r}-\mathrm{m}$ cross vein extending to the proximal part of $\mathrm{m} 2$ which layers and crosses the vein M2; distal pale spot in $\mathrm{r} 3$ larger than the distal pale spot in $\mathrm{m} 1$. Eyes bare and separated narrowly. Sensilla coeloconica present on all flagellomeres 1-13. Two functional spermathecae unequal and one rudimentary; absence of sclerotized ring at the end of the spermathecal duct. Middle leg with spines on tarsal segments 1 to 4 . This species is reported from the Balkans [63], Czech Republic, Denmark, Estonia, France, Germany, Hungary, Italy, Lithuania, Morocco, the Netherlands, Poland, Portugal, Russia, Slovakia, Spain, Turkey, Ukraine [64] and the UK. It is the only Palaearctic Culicoides with the pale spot on the R-M cross vein extending to the proximal part of $\mathrm{m} 2$ cell, which layers and crosses the M2 vein and therefore reaches the proximal part of $\mathrm{m} 1$ cell.

We report the presence of Culicoides (Sensiculicoides) univittatus Vimmer (synonym Culicoides agathensis Callot, Kremer \& Rioux, 1963). Females may be identified using the following criteria. Spotted wing as represented in Fig. 11; second radial cell entirely dark with the R3 vein slightly covered by the poststigmatic pale spot; pale spots on the distal part of $\mathrm{r} 3$ and $\mathrm{m} 1$ rounded and separated from the wing margin. Eyes bare and separated narrowly. Sensilla coeloconica present on all flagellomeres. Two functional spermathecae unequal and one rudimentary. Male wing similar to female with the usual sexual differences. Aedeagus with rounded basal arch extending about one-third of total length, distal process long (twothirds of total length), triangular with blunt rounded tip.

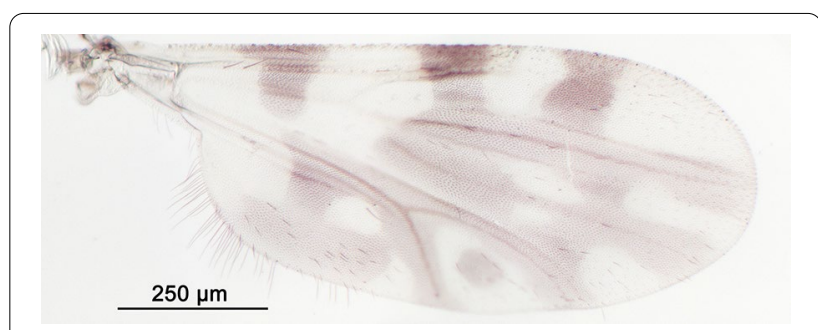

Fig. 8 Wing of Culicoides (Culicoides) paradoxalis Ramilo \& Delécolle 


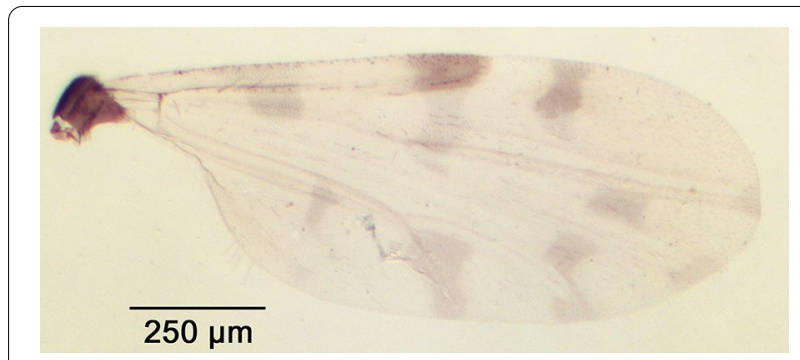

Fig. 9 Wing of Culicoides (Sensiculicoides) shaklawensis Khalaf

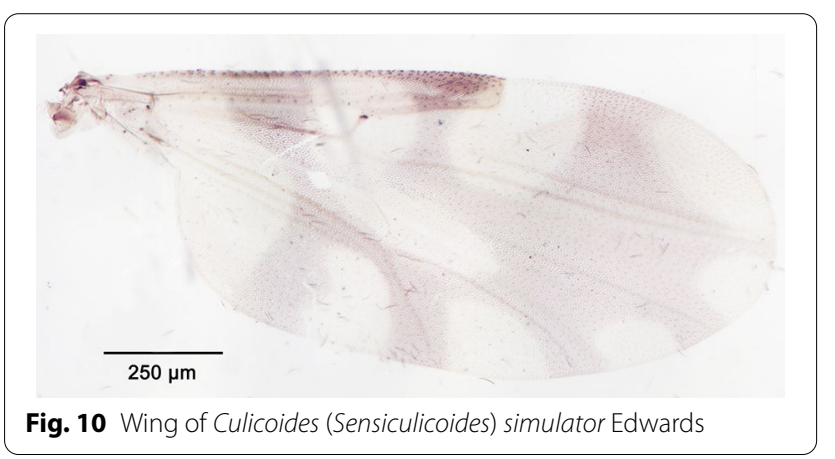

Parameres separated and tapering to a fine point; ninth sternite with ventral membrane bare or rarely bearing few spicules. This species is reported from Albania, Cyprus [61], France (southern mainland and Corsica), Italy (mainland and Sardinia), Morocco [11, 12], Portugal, Spain, Tunisia [20] and Turkey. This is the only species of Sensiculicoides with the pale spots on the distal part of $\mathrm{r} 3$ and $\mathrm{m} 1$ separated from the wing margin. Very close to C. pictipennis (Staeger), the male can be distinguished by the wing pattern and the distal process of the aedeagus, which is triangular for $C$. univittatus and rectangular for C. pictipennis.

We report the presence of Culicoides (Silvaticulicoides) achrayi Kettle \& Lawson. Females may be identified using the following criteria. Greyish wing with few spots as represented in Fig. 12; second radial cell completely dark with the R3 vein slightly covered by the post-stigmatic pale spot. Eyes bare and separated narrowly. Sensilla coeloconica present on flagellomeres 1, 9-13. Third palpal segment triangular and moderately swollen with multiple irregular sensory pits. Two functional spermathecae subequal with a long, pigmented neck and one rudimentary spermathecae; parallel sclerotized ring at the end of the spermathecal duct. Middle leg with spines on tarsal segments 1 to 4 . This species is reported from the Balkans [63], Belgium, Bulgaria, Cyprus, Czech Republic, Denmark, Estonia, France with Corsica, Germany, Hungary, Italy, Ireland,

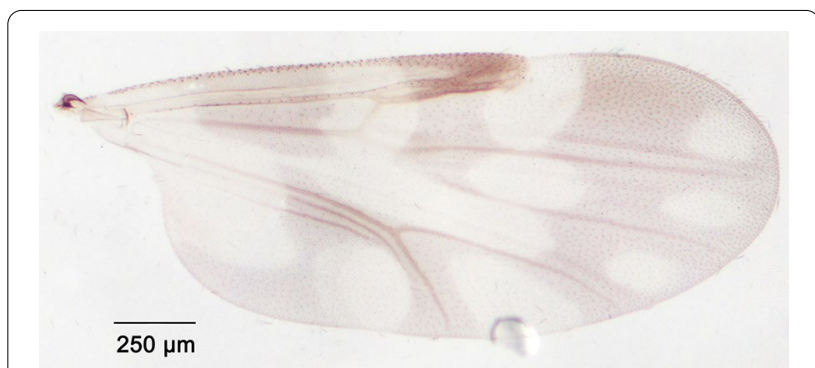

Fig. 11 Wing of Culicoides (Sensiculicoides) univittatus Vimmer

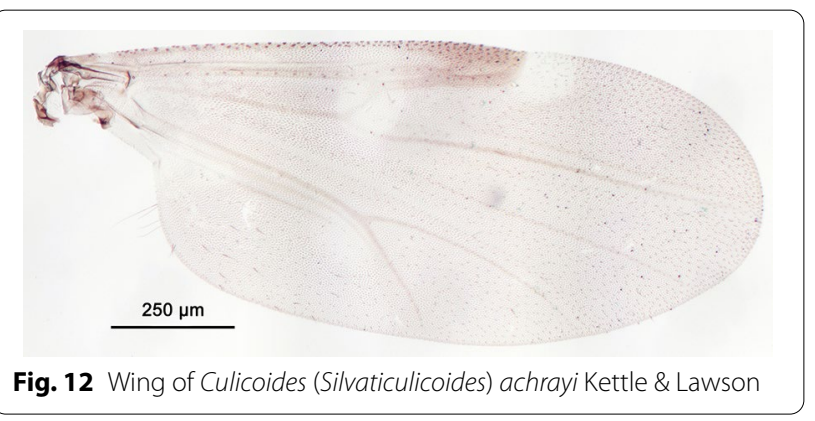

Lithuania, Morocco [65], the Near East, Poland, Portugal, Russia, Slovakia, Switzerland, Ukraine [64] and the UK.

We report the presence of Culicoides (Silvaticulicoides) picturatus Kremer \& Deduit. Females may be identified using the following criteria. Spotted wing as represented in Fig. 13; second radial cell completely dark, small and faint pale spots in the distal part of $\mathrm{r}$, $\mathrm{m} 1$ and $\mathrm{m} 2$ cells. Eyes bare and separated narrowly. Sensilla coeloconica present on flagellomeres 1, 9-13. Two functional spermathecae subequal and one rudimentary. Middle leg with spines on tarsal segments 1 to 3. Males may be identified using the following criteria. Wing similar to female with the usual sexual differences. Ninth sternite with broad and deep arch-shape caudomedian excavation, ventral membrane densely spiculate; basistyle with ventral root long and slender. This species was first described in France (Normandy region) by Kremer \& Deduit [66], and reported from Corsica, Denmark, Italy (mainland, Sardinia and Sicily), Morocco [11, 12], Portugal, Romania, Slovakia [67], Spain, Switzerland, Turkey, and the UK. Culicoides alazanicus Dzhafarov has a wing pattern similar to that of C. picturatus but can be easily separated from the latter under stereomiscrocopy by the ratio of the length of flagellomere 9 to that of flagellomere 8 on the female antenna being greater than 2. On the contrary, C. picturatus has such a ratio around 1.4 [67]. 


\section{Discussion}

We report ten new records of Culicoides species in Algeria (Table 2) by examining fresh material collected from November 2015 to September 2018 using light/suction traps in nine localities of the Tiaret region (northwestern Algeria). Among these ten species new to Algeria, $C$. shaklawensis, C. simulator and C. picturatus were already known from Morocco and C. univittatus from both Morocco and Tunisia [19, 20].

We also recorded the presence of $C$. chiopterus (one female) and $C$. dewulfi (13 females). Both species are widespread in Europe, but rare in the Mediterranean area. This is the first record of these species in North Africa, which underlines the need for further investigations to clarify their distribution in Algeria and more widely in the Maghreb. Currently, six species of the subgenus Avaritia are reported in Algeria (Table 2).

Among these species, $C$. imicola is a proven BTV and AHSV vector species: it is a livestock and equid biting species; numerous isolations of both viruses have been made from field-collected individuals; and the entire cycle of transmission of both viruses has been experimentally reproduced $[68,69]$. Culicoides imicola can be considered a probable SBV vector, as the viral genome was recovered from field-collected females [70, 71]. Culicoides obsoletus, C. scoticus, C. chiopterus and C. dewulfi are probable BTV and SBV vectors because of their ecological habits, and virus isolation or viral genome detection from field-collected individuals and experimental infections. BTV has been isolated from field-collected females, reported as ' $C$. obsoletus' taxon [72-74]; however, it was not clear whether this taxon referred to a species or an assemblage of species. BTV-8 genome has been identified from $C$. dewulfi and $C$. chiopterus fieldcollected individuals by real-time RT-PCR in the Netherlands [75, 76] and France [77]. BTV-1 genome was detected by real-time RT-PCR in C. obsoletus/C. scoticus parous females in the Basque country [78], and in $C$. obsoletus and C. scoticus parous females in Sardinia [79].

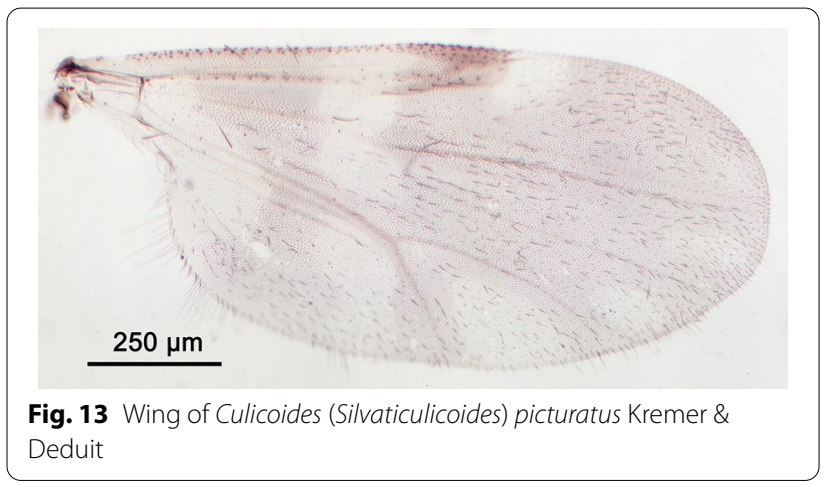

SBV genome has been identified in field-collected individuals: entire females of C. obsoletus/C. scoticus in Italy, the Netherlands and Poland [80-82] and of C. obsoletus, C. scoticus, C. dewulfi and C. chiopterus in France [70], and heads of $C$. obsoletus, $C$. scoticus, $C$. chiopterus and C. dewulfi in Belgium, Denmark and/or the Netherlands [83-85]. Moreover, C. obsoletus and C. scoticus from the UK have been experimentally infected with BTV-8 and BTV-9; C. scoticus exhibiting higher viral titers [86]. Dissemination efficiency has been estimated to be about $20 \%$ for BTV-1 in C. scoticus populations in Switzerland [32].

A total of 287 females and 1 male collected from the 'wilahya' of Tiaret were identified as C. grisescens. This species has been reported to be widely distributed in the northern part of the western Palaearctic region, but has never been recorded in North Africa. This species does not seem an efficient experimental vector for BTV [32]. We reported the presence of $C$. paradoxalis (1 female). This species has recently been described in southeastern France, Corsica and Portugal [45]. This new record confirms the Mediterranean distribution of C. paradoxalis. Currently, seven species of the subgenus Culicoides are reported in Algeria (Table 1). Among these, $C$ newsteadi, C. pulicaris and C. punctatus are considered possible BTV and SBV vectors, due to their feeding habits and to virus isolation or viral genome detections from field-collected individuals. In Italy, BTV has been isolated from C. pulicaris [87]; however, it was not clear whether this taxon referred to a species or an assemblage of species. BTV genome has been detected in field-collected individuals of $C$. newsteadi, C. pulicaris and C. punctatus in Spain and Italy [30, 33, 78, 79], while SBV genome has been detected in C. punctatus in Poland [82] and C. newsteadi and C. pulicaris in France [70].

We reported the presence of $C$. navaiae (286 females and 1 male), first described in Saudi Arabia: females by Lane [50] and males by Boorman [51]. This species of the subgenus Beltranmyia was relatively common in Saudi Arabia [88, 89], reported in the Sinai, Egypt [52], and recorded for the first time in our study in the Maghreb. When studying specimens from Algeria, Szadziewski [28] described a single female under the label C. (Beltranmyia) sp. indet. aff. homochrous because identification remained unsatisfactory. With the exception of the presence of sensilla coeloconica on the flagellomere 2 of the latter unidentified female [28], all characters are in agreement with the descriptions of $C$. navaiae females [50,52] and with our observations. Consequently, we agreed with Boorman's remark [51] that the female C. (Beltranmyia) sp. indet. aff. homochrous from Szadziewski [28] referred probably to $C$. navaiae. 
Table 2 Culicoides species list of Algeria

\begin{tabular}{|c|c|c|c|c|}
\hline Subgenus & Species & $\begin{array}{l}\text { Additional } \\
\text { records from } \\
\text { our data }\end{array}$ & $\begin{array}{l}\text { Proposed } \\
\text { checklist }\end{array}$ & References \\
\hline \multirow[t]{6}{*}{ Avaritia Fox, 1955} & Culicoides chiopterus Meigen, 1830 & $x$ & $\times$ & Present study \\
\hline & Culicoides dewulfi Goetghebuer, 1936 & $\times$ & $\times$ & Present study \\
\hline & Culicoides imicola Kieffer, 1913 & & $\times$ & {$[28,36,38-42]$} \\
\hline & Culicoides montanus Shakirzjanova, 1962 & & $\times$ & {$[39]$} \\
\hline & Culicoides obsoletus Meigen, 1818 & & $\times$ & {$[22,24,25,28,36,40-42]^{a}$} \\
\hline & Culicoides scoticus Downes \& Kettle, 1952 & & $\times$ & {$[28,36,41,42]$} \\
\hline \multirow[t]{3}{*}{ Beltranmyia Vargas, 1953} & Culicoides circumscriptus Kieffer, 1918 & & $\times$ & {$[25,27,28,36,40-42]$} \\
\hline & Culicoides navaiae Lane, 1983 & $\times$ & $x$ & Present study \\
\hline & Culicoides sphagnumensis Williams, 1955 & & & {$[42]$} \\
\hline \multirow[t]{6}{*}{ Culicoides Latreille, 1809} & Culicoides fagineus Edwards, 1939 & & $x$ & {$[25,41,42]$} \\
\hline & Culicoides grisescens Edwards, 1939 & $\times$ & $\times$ & Present study \\
\hline & Culicoides newsteadi Austen, 1921 & & $\times$ & {$[28,36,40-42]^{b}$} \\
\hline & Culicoides paradoxalis Ramilo \& Delécolle, 2013 & $\times$ & $x$ & Present study \\
\hline & Culicoides pulicaris (Linnaeus, 1758) & & $\times$ & {$[28,41,42]$} \\
\hline & Culicoides punctatus (Meigen, 1804) & & $\times$ & {$[28,36,41,42]$} \\
\hline \multirow[t]{2}{*}{ Monoculicoides Khalaf, 1954} & Culicoides parroti Kieffer, 1922 & & $x$ & {$[22,23,36]$} \\
\hline & Culicoides puncticollis (Becker, 1903) & & $\times$ & {$[22,23,25,28,36,40-42]^{c}$} \\
\hline \multirow[t]{10}{*}{ Oecacta Poey, 1853} & Culicoides azerbajdzhanicus Dzhafarov, 1962 & & $\times$ & {$[28,37]$} \\
\hline & $\begin{array}{l}\text { Culicoides corsicus Kremer, Leberre \& Beaucournu-Saguez, } \\
1971\end{array}$ & & $\times$ & [36] \\
\hline & Culicoides longipennis Khalaf, 1957 & & $x$ & {$[27,36]$} \\
\hline & Culicoides marcleti Callot, Kremer \& Basset, 1968 & & $\times$ & {$[27,28]$} \\
\hline & Culicoides ravus de Meillon, 1936 & & $x$ & {$[37]$} \\
\hline & Culicoides sahariensis Kieffer, 1923 & & $x$ & {$[23,25,28,36,40,42]^{d}$} \\
\hline & Culicoides santonicus Callot, Kremer, Rault \& Bach, 1966 & & $\times$ & [28] \\
\hline & Culicoides semimaculatus Clastrier, 1958 & & $\times$ & {$[26]$} \\
\hline & Culicoides sergenti Kieffer, 1921 & & $\times$ & {$[21,23,28,37]^{e}$} \\
\hline & Culicoides truncorum Edwards, 1939 & & & {$[42]$} \\
\hline \multirow[t]{2}{*}{ Pontoculicoides Remm, 1968} & Culicoides saevus Kieffer, 1922 & & $\times$ & {$[22-24,28,36,41,42]$} \\
\hline & Culicoides sejfadinei Dzhafarov, 1958 & & $x$ & {$[28]$} \\
\hline \multirow[t]{2}{*}{ Remmia Glukhova, 1977} & Culicoides kingi Austen, 1912 & & $\times$ & {$[28,37,40-42]$} \\
\hline & Culicoides schultzei (Enderlein, 1908) & & $\times$ & {$[25,42]$} \\
\hline \multirow[t]{16}{*}{ Sensiculicoides Shevchenko, 1977} & Culicoides begueti Clastrier, 1957 & & $\times$ & {$[25]$} \\
\hline & Culicoides cataneii Clastrier, 1957 & & $\times$ & {$[25,28,36,40]$} \\
\hline & Culicoides clastrieri Callot, Kremer \& Deduit, 1962 & & $x$ & {$[39,41,42]$} \\
\hline & Culicoides duddingstoni Kettle \& Lawson, 1955 & & $\times$ & {$[42]$} \\
\hline & Culicoides dzhafarovi Remm, 1967 & & $x$ & [28] \\
\hline & Culicoides festivipennis Kieffer, 1914 & & $\times$ & {$[36]$} \\
\hline & Culicoides gejgelensis Dzhafarov, 1964 & & $\times$ & {$[27,28,36]$} \\
\hline & Culicoides griseidorsum Kieffer, 1918 & & $\times$ & {$[28,41,42]$} \\
\hline & Culicoides heteroclitus Kremer \& Callot, 1965 & & $\times$ & {$[27,28,36]$} \\
\hline & Culicoides jumineri Callot \& Kremer, 1969 & & $x$ & {$[28,36,42]$} \\
\hline & Culicoides jurensis Callot, Kremer \& Deduit, 1962 & & & [42] \\
\hline & Culicoides kibunensis Tokunaga, 1937 & & $\times$ & {$[27]^{f}$} \\
\hline & Culicoides kurensis Dzhafarov, 1960 & & $x$ & [36] \\
\hline & Culicoides langeroni Kieffer, 1921 & & $\times$ & {$[28,37,40]$} \\
\hline & Culicoides maritimus Kieffer, 1924 & & $x$ & {$[28,41,42]$} \\
\hline & Culicoides odiatus Austen, 1921 & & $\times$ & {$[28,36]$} \\
\hline
\end{tabular}


Table 2 (continued)

\begin{tabular}{|c|c|c|c|c|}
\hline Subgenus & Species & $\begin{array}{l}\text { Additional } \\
\text { records from } \\
\text { our data }\end{array}$ & $\begin{array}{l}\text { Proposed } \\
\text { checklist }\end{array}$ & References \\
\hline & Culicoides pictipennis (Staeger, 1839) & & $\times$ & {$[28,41,42]$} \\
\hline & Culicoides poperinghensis Goetghebuer, 1953 & & $\times$ & {$[28]$} \\
\hline & Culicoides pseudopallidus Khalaf, 1961 & & $\times$ & {$[27,28,36]$} \\
\hline & Culicoides shaklawensis Khalaf, 1957 & $\times$ & $\times$ & Present study \\
\hline & Culicoides simulator Edwards, 1939 & $\times$ & $\times$ & Present study \\
\hline & Culicoides univittatus Vimmer, 1932 & $x$ & $x$ & Present study \\
\hline \multirow[t]{4}{*}{ Silvaticulicoides Glukhova, 1977} & Culicoides achrayi Kettle \& Lawson, 1955 & $\times$ & $x$ & Present study \\
\hline & Culicoides fascipennis (Staeger, 1839) & & $x$ & [36] \\
\hline & Culicoides picturatus Kremer \& Deduit, 1961 & $x$ & $\times$ & Present study \\
\hline & Culicoides subfasciipennis Kieffer, 1919 & & $\times$ & {$[28]$} \\
\hline Wirthomyia Vargas, 1973 & Culicoides faghihi Navai, 1971 & & $\times$ & [28] \\
\hline \multirow[t]{4}{*}{ Miscellaneous unplaced species } & Culicoides algeriensis Clastrier, 1957 & & $\times$ & [25] \\
\hline & Culicoides foleyi Kieffer, 1922 & & $\times$ & [22] \\
\hline & Culicoides nudipennis Kieffer, 1922 & & $\times$ & [22] \\
\hline & Culicoides paolae Boorman, Mellor \& Scaramozzino, 1996 & & $x$ & {$[36]$} \\
\hline
\end{tabular}

a Reported as Culicoides kabyliensis n. sp. Kieffer, 1922 in [22]

b Reported as Culicoides halophilus Kieffer, 1924 in [40]

c Reported as Culicoides distigma n. sp. Kieffer, 1922 and Culicoides donatieni n. sp. Kieffer, 1922 in [22]

d Reported as Culicoides coluzzii Callot, Kremer \& Bailly-Choumara, 1970 in [42]

e Reported as Culicoides citrinellus n. sp. Kieffer, 1923 in [23]

${ }^{f}$ Reported as Culicoides cubitalis Edwards, 1939 in [27]

References: [21] Kieffer (1921); [22] Kieffer (1922); [23] Kieffer (1923); [24] Goetghebuer (1939); [25] Clastrier (1957); [26] Clastrier (1958); [27] Callot et al. (1968); [28] Szadziewski (1984); [36] Baldet et al. (2003); [37] Baldet \& Delécolle (2006); [38] Nolan et al. (2008); [39] Djerbal \& Delécolle (2009); [41] Belkharchouche (2014); [42] Kabbout (2017); [40] Berrayah et al. (2020)

We collected three females identified as C. achrayi. This species has a wide distribution in the western Palaearctic region, including Mediterranean areas. This species has been collected in 2017 in Morocco as the first record in North Africa [65].

Kabbout [42] reported the first records of C. jurensis, C. sphagnumensis and C. truncorum from Algeria. As these species are rare and exhibit a non-Mediterranean Palaearctic distribution, we suggest not validating these species in the Culicoides checklist of Algeria until confirmation of identification by experts and molecular confirmation (Table 2).

The proposed checklist includes 59 Culicoides species in Algeria, while 54 species are recorded in Morocco [19] and 35 in Tunisia [20]. The Algerian fauna encompasses:

i. 28 species ( $47.5 \%$ of the species) with a wide and non-Mediterranean Palaearctic distribution: $C$. achrayi, C. begueti, C. chiopterus, C. circumscriptus, C. clastrieri, C. dewulfi, C. duddingstoni, C. fagineus, C. fascipennis, C. festivipennis, C. foleyi, C. grisescens, C. kibunensis, C. kurensis, C. montanus, C. navaiae, C. nudipennis, C. obsoletus, C. odiatus,
C. pictipennis, C. picturatus, C. pulicaris, C. punctatus, C. saevus, C. scoticus, C. shaklawensis, C. simulator and C. subfasciipennis;

ii. 28 species $(47.5 \%$ of the species) with a Mediterranean distribution: C. algeriensis, C. azerbajdzhanicus, C. cataneii, C. corsicus, C. dzhafarovi, C. faghihi, C. gejgelensis, C. griseidorsum, C. heteroclitus, C. jumineri, C. langeroni, C. longipennis, C. marcleti, C. maritimus, C. newsteadi, C. paolae, C. paradoxalis, C. parroti, C. poperinghensis, C. puncticollis, C. pseudopallidus, C. ravus, C. sahariensis, C. santonicus, C. sejfadinei, C. semimaculatus, C. sergenti and $C$. univittatus;

iii. 3 species (5.0\% of the species) with an Afrotropical distribution: C. imicola, C. kingi and C. schultzei.

Among these species, C. kingi is considered a probable vector of Onchocerca gutturosa affecting cattle in the Sahelian region $[90,91]$ and a potential vector of epizootic hemorrhagic disease virus [92, 93]. Recently, the detection of BTV genome in the head of C. circumscriptus and C. paoale in Sardinia has raised the question of 
the possible involvement of these species in BTV transmission in the Mediterranean basin [33].

\section{Conclusions}

The examination of fresh material resulted in the recording of ten new species for the Algerian fauna, although this material was collected in a small part of the country, namely in the 'wilahya' of Tiaret (northwestern Algeria). We have combined this information with existing published and grey literature to produce a comprehensive Culicoides checklist for Algeria of 59 species, including potential and probable vectors of arboviruses of veterinary interest. This is a prerequisite for the development of a barcode library and an atlas of diagnostic characters. Both may be useful for further ecological studies, in order to establish risk mapping for Culicoides-borne diseases in Algeria.

\begin{abstract}
Abbreviations
AHSV: African horse sickness virus; BTV: bluetongue virus; SBV: Schmallenberg virus; CIRAD: Centre de Coopération Internationale en Recherche Agronomique pour le Développement; IRD: Institut de Recherche pour le Développement; $\operatorname{cox} 1$ : cytochrome $c$ oxidase subunit 1; ITS2: internal transcribed spacer 2; RT-PCR: reverse transcription polymerase chain reaction; UV: ultraviolet; IIKC: interactive identification key for Culicoides; cua1: first cubital cell; CuA1: first cubital vein; m1: first median cell; m2: second median cell; M1: first median vein; $M 2$ : second median vein; $r 3$ : third radial cell; $R 3$ : third radial vein; $R-M$ : radial-median (cross vein).
\end{abstract}

\section{Acknowledgements}

We would like to thank Professor Szadziewski for helpful discussions on the data relating to the studies carried out in Algeria in the 1980s. We also thank the veterinary inspectors and the veterinarians of the agricultural subdivisions for their help in sample site selection, the Director and the staff of the equestrian center for help in collecting the biting midges, and the farmers for allowing the conducting of this study on their farms.

\section{Authors' contributions}

MB, SB and TBale designed the study. MB collected and sorted out the Culicoides specimens down to species, and wrote a draft of the paper. MD and IR provided valuable insights in morphological identifications, whereas BM confirmed the new species records, carried out molecular assays, revised the results and contributed to the discussion. TBald and TBale revised the manuscript, and made substantial improvements. All authors read and approved the final manuscript.

\section{Funding}

The Ministry of Higher Education and Scientific Research of Algeria supported, as part of the National Exceptional Program Scholarship (PNE 2018/2019), mobility of MB between the Ibn Khaldoun University of Tiaret (Algeria) and the CIRAD.

\section{Availability of data and materials}

The material is available on request and is held at the Institut de Parasitologie et de Pathologie Tropicale de Strasbourg (France) and at the Laboratoire de Biosystématique et Ecologie des Arthropodes, Faculté des Sciences de la Nature et de la Vie, University of Constantine 1 (Algeria).

Ethics approval and consent to participate Not applicable.

\section{Consent for publication}

Not applicable.

\section{Competing interests}

The authors declare that they have no competing interests.

\section{Author details}

${ }^{1}$ Ecole Nationale Supérieure de Biotechnologie, Taoufik Khaznadar, nouveau pôle universitaire Ali Mendjeli, B.P. E66, 25100 Constantine, Algérie. ${ }^{2}$ Faculté des Sciences de la Nature et de la Vie, Université Ibn Khaldoun, B.P.75 Zaaroura, Tiaret 1400, Algérie. ${ }^{3}$ Laboratoire de Biosystématique et Ecologie des Arthropodes, Faculté des Sciences de la Nature et de la Vie, Département de Biologie Animale, Université Frères Mentouri, Constantine 1 2500, Algérie. ${ }^{4}$ CIRAD, UMR ASTRE, 34398 Montpellier, France. ${ }^{5}$ Institut de Parasitologie et de Pathologies Tropicales de Strasbourg (IPPTS), UR 7292, 3 Rue Koeberlé, 67000 Strasbourg, France. ${ }^{6}$ ASTRE, University of Montpellier, CIRAD, INRAE, Montpellier, France. ${ }^{7}$ CIRAD, UMR ASTRE, 97491 Sainte-Clotilde, La Réunion, France. ${ }^{8}$ CIRAD, UMR ASTRE, 10101 Rabat, Morocco. ${ }^{9}$ Institut Agronomique et Vétérinaire Hassan II, Unité Microbiologie, Immunologie et Maladies Contagieuses, 10100 Rabat, Morocco.

Received: 17 June 2020 Accepted: 1 September 2020

Published online: 10 September 2020

\section{References}

1. Garros C, Balenghien T. Chapitre 14. Les culicoïdes (Diptera : Ceratopogonidae). In: Duvallet G, Fontenille D, Robert V, editors. Entomologie médicale et vétérinaire. Marseille: IRD Éditions \& Versailles: Éditions Quae; 2017.

2. Purse BV, Carpenter S, Venter GJ, Bellis G, Mullens BA. Bionomics of temperate and tropical Culicoides midges: knowledge gaps and consequences for transmission of Culicoides-borne viruses. Annu Rev Entomol. 2015;60:373-92.

3. Linley JR. Biting midges (Diptera: Ceratopogonidae) as vectors of nonviral animal pathogens. J Med Entomol. 1985;6:589-99.

4. Borkent A. The subgeneric classification of species of Culicoides 2016thoughts and a warning. 2016. https://www.inhs.illinois.edu/files/5014/ 6532/8290/CulicoidesSubgenera.pdf. Accessed 3 June 2020.

5. Campbell JA, Pelham-Clinton EC. Taxonomic review of the British species of Culicoides Latreille (Diptera, Ceratopogonidae). Proc R Soc Edinburgh B Biol. 1960;67:181-302.

6. Kremer M. Genre Culicoides Latreille. Paris: Lechavalier; 1965.

7. Delécolle JC. Nouvelle contribution à l'étude systématique et iconographique des espèces du genre Culicoides, (Diptéra: Cératopogonidae) du Nord-Est de la France. PhD thesis, Université Louis Pasteur de Strasbourg I, France; 1985

8. Mathieu B, Cêtre-Sossah C, Garros C, Chavernac D, Balenghien T, Carpenter $\mathrm{S}$, et al. Development and validation of IIKC: an interactive identification key for Culicoides (Diptera: Ceratopogonidae) females from the western Palaearctic region. Parasit Vectors. 2012;5:137.

9. Khamala CM, Kettle DS. The Culicoides Latreille (Diptera: Ceratopogonidae) of East Africa. Trans R Ent Soc Lond. 1971;123:1-95.

10. Glick J. Culicoides biting midges (Diptera: Ceratopogonidae) of Kenya. J Med Entomol. 1990;27:85-195.

11. Bailly-Choumara $H$, Kremer M. Deuxième contribution à l'étude des Culicoides du Maroc (Diptera: Ceratopogonidae). Entomol Méd Parasitol. 1970;4:383-91.

12. Kremer $M$, Hommel M, Bailly-Choumara H. Troisième contribution à l'étude faunistique des Culicoides du Maroc. Ann Parasitol Hum Comp. 1971;46:661-70.

13. Kremer M, Delécolle JC, Bailly-Choumara H, Chaker E. Cinquième contribution à l'étude faunistique des Culicoides (Diptera, Ceratopogonidae). Entom Méd Parasitol. 1979;3:195-9.

14. Chaker $\mathrm{E}$, Bailly-Choumara $\mathrm{H}$, Kremer M. Sixième contribution à l'étude faunistique des Culicoides du Maroc (Diptera, Cefratopogonidae). Bull Inst Sci Rabat. 1979;4:81-6.

15. Baylis M, Hasnaoui HE, Bouayoune $H$, Touti J, Mellor PS. The spatial and seasonal distribution of African horse sickness and its potential Culicoides vectors in Morocco. Med Vet Ent. 1997;11:203-12.

16. Bouayoune $H$, Touti J, El Hasnaoui H, Baylis M, Mellor PS. The Culicoides vectors of African horse sickness virus in Morocco: distribution and epidemiological implications. Arch Virol. 1998;14:113-25. 
17. Chaker E, Sfari M, Rabhi M, Rouis M, Babba H, Azaiez R. Note faunistique sur les Culicoides (Diptera, Ceratopogonidae) du Gouvernorat de Monastir (Tunisie). Parasite. 2005;12:359-61.

18. Slama D, Chaker E, Mathieu B, Babba H, Depaquit J, Augot D. Biting midges monitoring (Diptera: Ceratopogonidae: Culicoides Latreille) in the governate of Monastir (Tunisia): species composition and molecular investigations. Parasitol Res. 2014;113:2435-43.

19. Bourquia M, Garros C, Rakotoarivony I, Gardès L, Huber K, Boukhari I, et al. Update of the species checklist of Culicoides Latreille, 1809 biting midges (Diptera: Ceratopogonidae) of Morocco. Parasit Vectors. 2019;12:459.

20. Sghaier S, Hammami S, Goffredo M, Hammami M, Portanti O, Lorusso A, et al. New species of the genus Culicoides (Diptera: Ceratopogonidae) for Tunisia, with detection of bluetongue viruses in vectors. Vet Ital. 2017;53:357-66.

21. Kieffer J. Sur quelques Diptères piqueurs de la Tribu Ceratopogoninae. Arch Inst Pasteur Afr Nord. 1921;1:107-15.

22. Kieffer J. Nouveaux Chironomides piqueurs habitant l'Algérie. Arch Inst Pasteur Alger. 1922;2:494-518.

23. Kieffer J. Ceratopogonines recueillis au Sahara Constantinois. Arch Inst Pasteur Alger. 1923;4:654-83.

24. Goetghebuer M. Cératopogonides et Chironomides recueillis en Algérie. Bull Ann Soc R Belge Ent. 1939;79:59-62.

25. Clastrier J. Notes sur les Cératopogonidés. II. Quelques Culicoides d'Algérie à ailes tachetées. Arch Inst Pasteur Alger. 1957;35:404-44.

26. Clastrier J. Notes sur les Cératopogonidés. III. Culicoides semimaculatus n. sp. d'Algérie. Arch Inst Pasteur Alger. 1958;36:55-60.

27. Callot J, Kremer M, Basset M. Culicoides marcleti n. sp. et nouvelles localisations de culicoïdes (Diptères, Cératopogonidés) de la région méditerranéenne et particulièrement d'Algérie. Bull Soc Pathol Exot. 1968;61:271-82

28. Szadziewski R. Ceratopogonidae (Diptera) from Algeria. VI. Culicoides Latr. Pol Pismo Entomol. 1984;54:163-82.

29. Mellor PS, Hamblin C. African horse sickness. Vet Res. 2004;35:445-66.

30. Goffredo M, Catalani M, Federici V, Portanti O, Marini V, Mancini G, et al. Vector species of Culicoides midges implicated in the 2012-2014 bluetongue epidemics in Italy. Vet Ital. 2015;51:131-8.

31. Goffredo M, Meiswinkel R, Federici V, Di Nicola F, Mancini G, Ippoliti $C$, et al. The 'Culicoides obsoletus group' in Italy: relative abundance, geographic range, and role as vector for bluetongue virus. Vet Ital. 2016:52:235-41.

32. Paslaru AL, Mathis A, Torgerson P, Veronesi E. Vector competence of pre-alpine Culicoides (Diptera: Ceratopogonidae) for bluetongue virus serotypes 1, 4 and 8. Parasit Vectors. 2018;11:466.

33. Foxi C, Meloni G, Puggioni G, Manunta D, Rocchigiani A, Vento L, et al. Bluetongue virus detection in new Culicoides species in Sardinia. Italy. Vet Rec. 2019;184:621.

34. Cetre-Sossah C, Madani H, Sailleau C, Nomikou K, Sadaoui H, Zientara S, et al. Molecular epidemiology of bluetongue virus serotype 1 isolated in 2006 from Algeria. Res Vet Sci. 2011;91:486-97.

35. Kardjadj M. An epidemiological overview of small ruminant diseases in Algeria. Rev Sci Tech OIE. 2017;36:997-1006.

36. Baldet T, Albina E, Delécolle JC. Appui à la surveillance de la fièvre catarrhale ovine en Algérie. Montpellier: Cirad; 2003. p. 10.

37. Baldet T, Delécolle JC. Mission entomologique d'appui à la surveillance de la fièvre catarrhale ovine en Algérie. Montpellier: Cirad; 2006. p. 6.

38. Nolan DV, Dallas JF, Piertney SB, Mordue (Luntz) AJ. Incursion and range expansion in the bluetongue vector Culicoides imicola in the Mediterranean basin: a phylogeographic analysis. Med Vet Entomol. 2008;22:340-51.

39. Djerbal M, Delécolle JC. Entomological surveillance of bluetongue in Algeria. Rev Élev Méd Vét Pays Trop. 2009;62:81-180.

40. Berrayah H, Hwang HS, Lee KY. Molecular identification of Culicoides (Diptera: Ceratopogonidae) species in Algeria. Acta Trop. 2020;202:105261.

41. Belkharchouche M. Contribution à l'étude de la biodiversité des Culicoides (Diptera, Ceratopogonidae) responsable de la fièvre catarrhale dans la région Est-algerien foyer d'Oum-El-Bouaghi. MSc Thesis, Université d'Oum El Bouaghi, Algeria; 2014.

42. Kabbout N. Contribution à l'étude bio écologique des insectes d'intérêt médical dans le Nord-Est Algérien. PhD thesis, Université d'Oum El Bouaghi, Algeria; 2017.
43. Meddour R. Bioclimatologie, phytogéographie et phytosociologie en Algérie. Exemple des groupements forestiers et préforestiers de la Kabylie djurdjuréenne. PhD thesis, Université de Tizi Ouzou, Algeria; 2010.

44. Wirth WW, Marston N. A method for mounting small insects on microscope slides in Canada balsam. Ann Entomol Soc Am. 1968;61:783-4.

45. Ramilo D, Garros C, Mathieu B, Benedet C, Allène X, Silva E, et al. Description of Culicoides paradoxalis sp. nov. from France and Portugal (Diptera: (eratopogonidae). Zootaxa. 2013;3745:243.

46. Szadziewski R, Filatov S, Dominiak P. A redescription of Culicoides griseidorsum Kieffer, 1918, with comments on subgeneric position of some European taxa (Diptera: Ceratopogonidae). Zootaxa. 2016;4107:413-22.

47. Szadziewski R, Borkent A, Dominiak P. Fauna Europaea: Ceratopogonidae. 2001. In: Beuk P, Pape T, de Jong YSDM. Fauna Europaea: Diptera, Nematocera. Fauna Europaea version 2.6.2. 2013. https://www.fauna-eu.org. Accessed 3 June 2020.

48. Gutsevich AV. Biting midges of the genus Culicoides (Diptera, Heleidae) of the Ukrainian Carpathians (Transcarpathian province). Entomol Rev. 1964:43:310-4.

49. Schwenkenbecher JM, Mordue AJ, Piertney SB. Phylogenetic analysis indicates that Culicoides dewulfi should not be considered part of the Culicoides obsoletus complex. Bull Entomol Res. 2009;99:371-5.

50. Lane RP. Insects of Saudi Arabia Culicoides (Diptera: Ceratopogonidae) of Saudi Arabia and their potential veterinary importance. Fauna Saudi Arabia. 1983;5:529-44.

51. Boorman J. Culicoides (Diptera, Ceratopogonidae) of Arabian Peninsula with notes on their medical and veterinary importance. Fauna Saudi Arabia. 1989;10:66.

52. Kremer M, Delécolle JC, Braverman Y. A new and a redescription species of Culicoides from Sinai (Diptera, Ceratopogonidae). J Zool. 1991;37:151-7.

53. Remm H, Zhogolev DT. Contributions to the fauna of biting midges (Diptera, Ceratopogonidae) of the Crimea. Entomol Obozr. 1968;47:826-42.

54. Glukhova VM. Culicoides (Diptera, Ceratopogonidae) of Russia and adjacent lands. Int J Dipterol Res. 2005;16:1-77.

55. Nielsen OB. The biting midges of Lyngby Aamose (Culicoides: Ceratopogonidae). Aarhus: Naturhistorisk Museum; 1963.

56. Gomulski LM, Meiswinkel R, Delécolle JC, Goffredo M, Gasperi G. Phylogeny of the subgenus Culicoides and related species in Italy, inferred from internal transcribed spacer 2 ribosomal DNA sequences. Med Vet Entomol. 2006;20:229-38.

57. Meiswinkel R, Gomulski LM, Delécolle JC, Goffredo M, Gasperi G. The taxonomy of Culicoides vector complexes - unfinished business. Vet Ital. 2004:40:151-9.

58. Nielsen SA, Kristensen M. Delineation of Culicoides species by morphology and barcode exemplified by three new species of the subgenus Culicoides (Diptera: Ceratopogonidae) from Scandinavia. Parasit Vectors. 2015:8:151.

59. Sarvašová A, Kočišová A, Candolfi E, Mathieu B. Description of Culicoides bysta $n$. sp., a new member of the Pulicaris group (Diptera: Ceratopogonidae) from Slovakia. Parasit Vectors. 2017;10:279.

60. Talavera S, Muñoz-Muñoz F, Verdún M, Pagès Martinez N. Morphology and DNA barcoding reveal three species in one: description of Culicoides cryptipulicaris sp. nov. and Culicoides quasipulicaris sp. nov. in the subgenus Culicoides. Med Vet Entomol. 2017:31:178-91.

61. Boorman J. Culicoides (Diptera, Ceratopogonidae) from Cyprus. Cah ORSTOM. 1974:12:7-13.

62. Delécolle JC. Deux Culicoides nouveaux pour la faune de France: $C$. dendriticus Boorman et C. shaklawensis Khalaf (Diptera, Ceratopogonidae). Bull Ass Philomathique Alsace Lorraine. 1995;31:37-46.

63. Pudar D, Petrić D, Allène X, Alten B, Ayhan N, Cvetkovikj A, et al. An update of the Culicoides (Diptera: Ceratopogonidae) checklist for the Balkans. Parasit Vectors. 2018;11:462.

64. Shevchenko AK. Bloodsucking midges of the genus Culicoides (Diptera, Ceratopogonidae) from the valley of the middle current of the Desna. Entomol Obozr. 1967:46:163-79.

65. Bourquia M. Déterminants environnementaux de la distribution des Culicoides (Diptera : Ceratopogonidae), moucherons vecteurs de virus animaux d'intérêt économique, au Maroc. Ph.D. thesis, IAV Hassan II, Morocco and Montpellier SupAgro, France; 2019.

66. Kremer M, Deduit Y. Sur quelques Culicoides (Diptera: Ceratopogonidae) de Normandie. Description de Culicoides picturatus n. sp. Ann Parasitol Hum Comp. 1961;36:701-5. 
67. Sarvašová A, Goffredo M, Sopoliga I, Savini G, Kočišová A. Culicoides midges (Diptera: Ceratopogonidae) as vectors of orbiviruses in Slovakia. Vet Ital. 2014:50:203-12.

68. Du Toit RM. The transmission of blue-tongue and horse-sickness by Culicoides. Onderstepoort J Vet Sci Anim Ind. 1944;19:7-16.

69. Mellor PS, Boorman J, Baylis M. Culicoides biting midges: their role as arbovirus vectors. Annu Rev Entomol. 2000;45:307-40.

70. Balenghien T, Pagès N, Goffredo M, Carpenter S, Augot D, Jacquier E, et al. The emergence of Schmallenberg virus across Culicoides communities and ecosystems in Europe. Prev Vet Med. 2014;116:360-9.

71. Ségard A, Gardes L, Jacquier E, Grillet C, Mathieu B, Rakotoarivony I, et al. Schmallenberg virus in Culicoides Latreille (Diptera: Ceratopogonidae) populations in France during 2011-2012 outbreak. Transbound Emerg Dis. 2017:65:94-103.

72. Mellor PS, Pitzolis G. Observations on breeding sites and light-trap collections of Culicoides during an outbreak of bluetongue in Cyprus. Bull Entomol Res. 1979;69:229-34.

73. Savini G, Goffredo M, Monaco F, Di Gennaro A, De Santis P, Meiswinkel $\mathrm{R}$, et al. The isolation of bluetongue virus from field populations of the Obsoletus Complex in central Italy. Vet Ital. 2004:40:286-91.

74. De Liberato C, Scavia G, Lorenzetti R, Scaramozzino P, Amaddeo D, Cardeti $G$, et al. Identification of Culicoides obsoletus (Diptera: Ceratopogonidae) as a vector of bluetongue virus in central Italy. Vet Rec. 2005;156:301-4.

75. Meiswinkel R, Van Rijn P, Leijs P, Goffredo M. Potential new Culicoides vector of bluetongue virus in northern Europe. Vet Rec. 2007;161:564.

76. Dijkstra E, van der Ven IJK, Meiswinkel R, Holzel DR, van Rijn PA, Meiswinkel R. Culicoides chiopterus as a potential vector of bluetongue virus in Europe. Vet Rec. 2008;162:422.

77. Venail R, Balenghien T, Guis H, Tran A, Setier-Rio ML, Delécolle JC, et al. Assessing diversity and abundance of vector populations at a national scale: example of Culicoides surveillance in France after bluetongue virus emergence. In: Mehlhorn H, editor. Arthropods as vectors of emerging diseases. Heidelberg: Springer; 2012. p. 77-102.

78. Romon P, Higuera M, Delécolle JC, Baldet T, Aduriz G, Goldarazena A. Phenology and attraction of potential Culicoides vectors of bluetongue virus in Basque Country (northern Spain). Vet Parasitol. 2012;186:415-24.

79. Foxi C, Delrio G, Falchi G, Marche MG, Satta G, Ruiu L. Role of different Culicoides vectors (Diptera: Ceratopogonidae) in bluetongue virus transmission and overwintering in Sardinia (Italy). Parasit Vectors. 2016;9:440

80. Elbers ARW, Meiswinkel R, van Weezep E, van der Poel WHM, Kooi EA Schmallenberg virus in Culicoides spp. biting midges, the Netherlands, 2011. Emerg Infect Dis. 2013;19:04
81. Goffredo M, Monaco F, Capelli G, Quaglia M, Federici V, Catalani M, et al. Schmallenberg virus in Italy: a retrospective survey in Culicoides stored during the bluetongue Italian surveillance program. Prev Vet Med. 2013;111:230-6.

82. Larska M, Lechowski L, Grochowska M, Żmudziński JF. Detection of the Schmallenberg virus in nulliparous Culicoides obsoletus/scoticus complex and C. punctatus, the possibility of transovarial virus transmission in the midge population and of a new vector. Vet Microbiol. 2013;166:467-73.

83. De Regge N, Deblauwe I, De Deken R, Vantieghem P, Madder M, Geysen D, et al. Detection of Schmallenberg virus in different Culicoides spp. by real-time RT-PCR. Transbound Emerg Dis. 2012;59:471-5.

84. Rasmussen LD, Kristensen B, Kirkeby C, Rasmussen TB, Belsham GJ, Bødker $\mathrm{R}$, et al. Culicoides as vectors of Schmallenberg virus. Emerg Infect Dis. 2012;18:1204

85. Elbers ARW, Meiswinkel R, van Weezep E, Kooi EA, van der Poel WHM. Schmallenberg virus in Culicoides biting midges in the Netherlands in 2012. Transbound Emerg Dis. 2015;62:339-42.

86. Carpenter S, McArthur C, Selby R, Ward R, Nolan DV, Luntz AM, et al. Experimental infection studies of UK Culicoides species midges with bluetongue virus serotypes 8 and 9. Vet Rec. 2008;163:589-92.

87. Caracappa S, Torina A, Guercio A, Vitale F, Calabro A, Purpari G, et al. Identification of a novel bluetongue virus vector species of Culicoides in Sicily. Vet Rec. 2003;153:71-4.

88. Hilali M, Abu-Elzein ET, Al-Afaleq A, Boorman J, Alatyia S, Alnaeem A. Culicoides midges (Ceratopogonidae) in some localities of Saudi Arabia and their veterinary significance. Vet Arh. 2003;73:285-94.

89. Alahmed AM, Kheir SM, Al Khereiji MA. Distribution of Culicoides Latreille (Diptera: Ceratopogonidae) in Saudi Arabia. J Entomol. 2010;7:227-34.

90. El Sinnary K, Hussein HS. Culicoides kingi, Austen: a vector of Onchocerca gutturosa (Neumann, 1910) in the Sudan. Ann Trop Med Parasitol. 1980;74:655-6.

91. Bakhoum MT, Sarr M, Fall AG, Huber K, Fall M, Sembène M, et al. DNA barcoding and molecular identification of field-collected Culicoides larvae in the Niayes area of Senegal. Parasit Vectors. 2018;11:10.

92. Lee VH. Isolation of viruses from field populations of Culicoides (Diptera: Ceratopogonidae) in Nigeria. J Med Entomol. 1979;16:76-9.

93. Mellor PS, Osborne R, Jennings DM. Isolation of bluetongue and related viruses from Culicoides spp. in the Sudan. Epidemiol Infect. 1984;93:621-8.

\section{Publisher's Note}

Springer Nature remains neutral with regard to jurisdictional claims in published maps and institutional affiliations.
Ready to submit your research? Choose BMC and benefit from:

- fast, convenient online submission

- thorough peer review by experienced researchers in your field

- rapid publication on acceptance

- support for research data, including large and complex data types

- gold Open Access which fosters wider collaboration and increased citations

- maximum visibility for your research: over $100 \mathrm{M}$ website views per year

At BMC, research is always in progress.

Learn more biomedcentral.com/submissions 